\title{
HIPK4 is essential for murine spermiogenesis
}

\section{J. Aaron Crapster, ${ }^{a}$ * Paul G. Rack, ${ }^{a}$ Zane J. Hellmann, ${ }^{a}$ Joshua E. Elias, ${ }^{b}$ John J. Perrino, ${ }^{c}$} Barry Behr, ${ }^{d}$ Yanfeng Li, ${ }^{e}$ Jennifer Lin, ${ }^{e}$ Hong Zeng, ${ }^{e}$ and James K. Chen ${ }^{a, f *}$

a Department of Chemical and Systems Biology, Stanford University School of Medicine, Stanford, CA

b Chan Zuckerberg Biohub, Stanford University, Stanford, CA

c Cell Science Imaging Facility, Stanford University School of Medicine, Stanford, CA

${ }^{d}$ Department of Obstetrics and Gynecology, Reproductive Endocrinology and Infertility, Stanford University School of Medicine, Stanford, CA

e Transgenic, Knockout, and Tumor Model Center, Stanford University School of Medicine, Stanford, CA

$f$ Department of Developmental Biology, Stanford University School of Medicine, Stanford, CA

* Correspondence should be addressed to J.A.C. (aaron.crapster@vibliome.com) or J.K.C. (jameschen@stanford.edu) 


\section{ABSTRACT}

Mammalian spermiogenesis is a remarkable cellular transformation, during which round spermatids elongate into chromatin-condensed spermatozoa. The signaling pathways that coordinate this process are not well understood, and we demonstrate here that homeodomain-

37 interacting protein kinase 4 (HIPK4) is essential for spermiogenesis and male fertility in mice.

38 HIPK4 is predominantly expressed in round and early elongating spermatids, and Hipk4 knockout 39 males are sterile, exhibiting phenotypes consistent with oligoasthenoteratozoospermia. Hipk4

40 mutant sperm have reduced oocyte binding and are incompetent for in vitro fertilization, but they

41 can still produce viable offspring via intracytoplasmic sperm injection. Ultrastructural analyses of

42 HIPK4-null male germ cells reveal defects in the filamentous actin (F-actin)-scaffolded

43 acroplaxome during spermatid elongation and abnormal head morphologies in mature

44 spermatozoa. We further observe that HIPK4 overexpression induces branched F-actin structures

45 in cultured fibroblasts, supporting a role for this kinase in cytoskeleton remodeling. Our findings

46 establish HIPK4 as an essential regulator of sperm head shaping and potential target for male

47 contraception. 


\section{INTRODUCTION}

Spermiogenesis is a critical, post-meiotic phase of male gametogenesis defined by the

51 differentiation of spermatids into spermatozoa (Figure 1A-B) (Russell, et al., 1990). This dramatic

52 morphological transformation is mediated by a series of cytological processes that are unique to

53 the testis (Kierszenbaum, et al., 2007; O'Donnell, 2014). In round spermatids, Golgi-derived

54 vesicles give rise to the acrosome (Berruti, et al., 2011), a cap-like structure that is anchored to

55 the anterior nuclear membrane by a filamentous actin (F-actin)- and keratin-containing plate

56 called the acroplaxome (Kierszenbaum, et al., 2003; Kierszenbaum, et al., 2004). Neighboring

57 Sertoli cells form an apical specialization that circumscribes each spermatid head, and F-actin

58 hoops within these anchoring junctions apply external forces to the acrosome-acroplaxome

59 complex and underlying spermatid nucleus (Wong, et al., 2008). The posterior nuclear pole in

60 spermatids is simultaneously girdled by a transient microtubule- and F-actin-scaffolded structure

61 called the manchette, which extends from the basal body of the developing flagellum and is

62 separated from the acrosome-acroplaxome complex by a narrow groove (Lehti, et al., 2016).

As spermiogenesis proceeds, these membranous and cytoskeletal structures act in concert

to elongate the spermatid head. The spermatid nucleus becomes highly compact as chromatin condenses into a quiescent state (Rathke, et al., 2014), and cytoplasmic contents of the germ cell are also expelled through residual bodies or intercellular bridges to Sertoli cells (Sprando, et al., 1987; Zheng, et al., 2007). The acrosome-acroplaxome complex and manchette concurrently mediate protein transport from the Golgi to the developing flagellum, delivering cargoes required

69 for flagellum assembly and function(Kierszenbaum, et al., 2011; Kierszenbaum, et al., 2004). In

70 mature sperm, the acrosome then promotes sperm-egg fusion through the exocytotic release of

71 digestive enzymes and the display of oocyte-binding receptors that are localized to the inner

72 acrosomal membrane (Stival, et al., 2016).

73 In contrast to these detailed cytological descriptions, our understanding of the molecular

74 mechanisms that coordinate spermiogenesis is still nascent. Initial insights into this process have 
bioRxiv preprint doi: https://doi.org/10.1101/703637; this version posted July 16, 2019. The copyright holder for this preprint (which was not certified by peer review) is the author/funder, who has granted bioRxiv a license to display the preprint in perpetuity. It is made available under aCC-BY-NC-ND 4.0 International license.

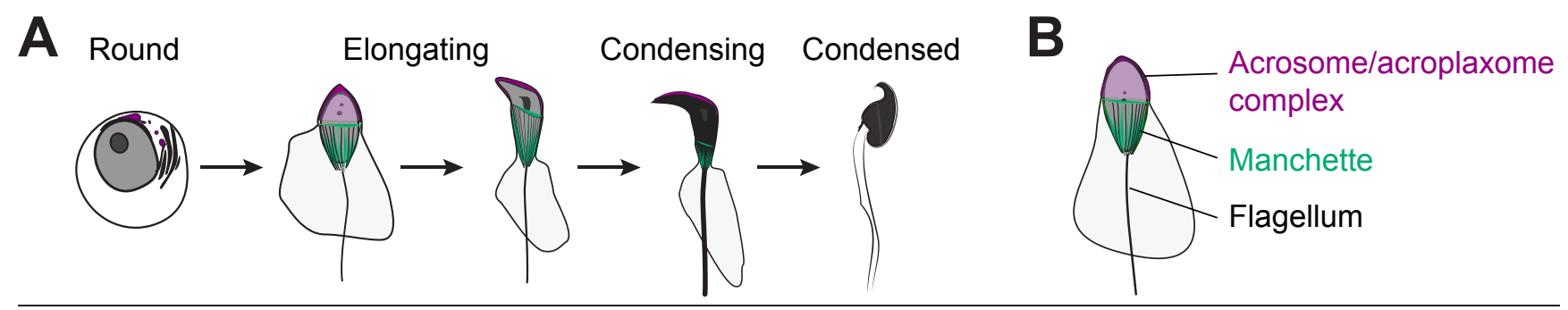

Figure 1. Key steps of spermiogenesis. (A) Schematic representation of murine male germ cells transitioning from round spermatids to condensed spermatoø a. These steps occur within the testis seminiferous epithelium and are conserved in all mammals. (B) Illustration of an elongating spermatid highlighting structural features that are established during spermiogenesis. 
75 been provided by mouse mutants with spermatogenic and male fertility defects (de Boer, et al.,

76 2015; Yan, 2009). For example, pro-acrosomal vesicles fail to fuse in mice that lack the

77 nucleoporin-like protein HRB/AGFG1 (Kang-Decker, et al., 2001; Kierszenbaum, et al., 2004),

78 nuclear membrane protein DPY19L2 (Pierre, et al., 2012), certain Golgi-associated proteins

79 [GOPC (Yao, et al., 2002), PICK1 (Xiao, et al., 2009), and GM130 (Han, et al., 2017)], or

80 acrosomal factors [SPACA1 (Fujihara, et al., 2012) and SPATA16 (Fujihara, et al., 2017)]. These

81 mutants consequently produce acrosome-less sperm with rounded heads-defects that are

82 characteristic of globozoospermia. Acrosome biogenesis also requires the matrix component

83 ACRBP (Kanemori, et al., 2016) and the coiled coil protein CCDC62 (Li, Y., et al., 2017; Pasek,

84 et al., 2016), and loss of either acrosomal protein can cause phenotypes resembling

85 oligoasthenoteratozoospermia (OAT), a fertility disorder characterized by low sperm

86 concentrations and spermatozoa with abnormal shapes and reduced motility (Tuttelmann, et al.,

87 2018).

88 Murine models have similarly revealed proteins that are required for manchette and

89 flagellum assembly, including the RIMBP3-HOOK1 (Zhou, et al., 2009), LRGUK1-HOOK2 (Liu,

90 et al., 2015), MEIG1-PACRG-SPAG16L (Li, W., et al., 2015), and FU (Nozawa, et al., 2014).

91 Manchette shaping and degradation are also essential for sperm development, as demonstrated

92 by the OAT-like phenotypes of mice expressing a loss-of-function variant of the microtubule-

93 severing protein Katanin p80 (O'Donnell, et al., 2012). As spermiogenesis proceeds,

94 membranous and cytoskeletal structures are dynamically coupled by distinct LINC (Linker of

95 Nucleoskeleton and Cytoskeleton) complexes. These include LINC components that reside in the

96 outer acrosomal membrane (SUN1 and nesprin3) (Gob, et al., 2010) or posterior nuclear

97 envelope (SUN3, SUN4, SUN5, and nesprin1) (Gob, et al., 2010; Pasch, et al., 2015; Shang, Y.,

98 et al., 2017). For example, loss of SUN4 function in mice causes manchette disorganization,

99 sperm head defects, and male sterility. 
Factors that specifically contribute to acroplaxome function have been more difficult to identify and study. Actin-binding proteins such as myosins Va and VI, profilins III and IV, and cortactin localize to the acroplaxome and have been implicated in its regulation (Behnen, et al., 2009; Kierszenbaum, et al., 2003; Kierszenbaum, et al., 2008; Kierszenbaum, et al., 2011;

Zakrzewski, et al., 2017); however, their wide-spread expression in somatic tissues has hindered

105 functional studies. One notable exception is the actin capping protein, CAPZA3, a spermatid106 specific factor that associates with CAPZB3 and binds to the barbed ends of F-actin. Capza3 107 mutant male mice are sterile and have OAT-like defects, indicating that F-actin dynamics within 108 the acroplaxome play an important role in spermiogenesis (Geyer, et al., 2009).

Upstream signaling proteins that control cytoskeletal dynamics are likely to be critical drivers

of spermatid differentiation. For instance, PLC $\gamma-1$ phosphorylation is dysregulated in the germ cells of $\mathrm{KIT}^{\mathrm{D} 814 \mathrm{Y}}$ mutant mice, leading to mislocalized manchettes and deformed spermatid heads (Schnabel, et al., 2005). Phosphoproteomic analyses indicate that several kinase-dependent pathways are active throughout sperm development, but the roles of specific kinases in spermiogenesis are not well understood (Castillo, et al., 2019). Here we describe an essential function for homeodomain-interacting protein kinase 4 (HIPK4) in murine spermiogenesis and fertility. This dual-specificity kinase is predominantly expressed in the testis, where it is restricted

117 to round and early elongating spermatids. Male Hipk4 knockout mice are sterile and exhibit 118 spermatogenic defects characteristic of OAT. Sperm produced by these mutant mice are also 119 incompetent for oocyte binding and in vitro fertilization, and they exhibit head defects associated 120 with dysregulation of the acrosome-acroplaxome complex. Consistent with these observations, 121 HIPK4 overexpression in cultured somatic cells remodels the F-actin cytoskeleton and alters the 122 phosphorylation state of multiple actin-interacting proteins. Taken together, our studies 123 demonstrate that HIPK4 regulates acrosome-acroplaxome dynamics, spermatid head shaping, and ultimately, sperm function. 
126

127

128

129

130

\section{RESULTS}

HIPK4 is expressed in differentiating spermatids

Gene expression data available through the Genotype Tissue Expression Project (https://www.gtexportal.org) and the Mammalian Reproductive Genetics Database (http://mrgd.org) indicate that HIPK4 is largely expressed in the testis, with lower levels detected in the brain. Using a tissue cDNA array and quantitative PCR, we also found that Hipk4 is robustly transcribed in the adult murine testis (Figure 2A). In situ hybridization of testis sections obtained from 8-week-old C57BL/6NJ mice revealed that Hipk4 is transcribed specifically in round and early elongating spermatids (Figure 2B), and we observed comparable HIPK4 expression patterns in adult human testis samples (Figure 2C). We then assayed testis sections from juvenile male mice of different ages to determine precisely when Hipk4 is expressed during spermatogenesis, taking advantage of the initial, synchronized wave of male germ cell development. Hipk4 transcripts were first detected in germ cells at 21 days postpartum (dpp), coinciding with the appearance of step 2-3 round spermatids (Figure 2 - figure supplement 1 ). The population of Hipk4-positive spermatids expanded until $29 \mathrm{dpp}$, at which point Hipk4 mRNA became undetectable in elongating spermatids circumscribing the seminiferous lumen. These results suggest that HIPK4 specifically functions within male germ cells as they transition from round to elongating spermatids.

\section{HIPK4 is essential for male fertility}

Hipk4 knockout mice were first reported in a 2008 patent application by Bayer Schering Pharma, which described general defects in sperm morphology and number (Sacher, et al., 2008). However, the fertility of these mutant mice was not characterized, nor were the mice made publicly available. As part of the Knockout Mouse Phenotyping Program (KOMP ${ }^{2}$ ), the Jackson Laboratory generated mice containing a Hipk4 null allele $(\operatorname{tm} 1 b)$, in which a $\beta$-galactosidase reporter replaces exons 2 and 3 . We established a colony of Hipk $4^{\operatorname{tm} 1 b / t m 1 b}$ mice (henceforth 


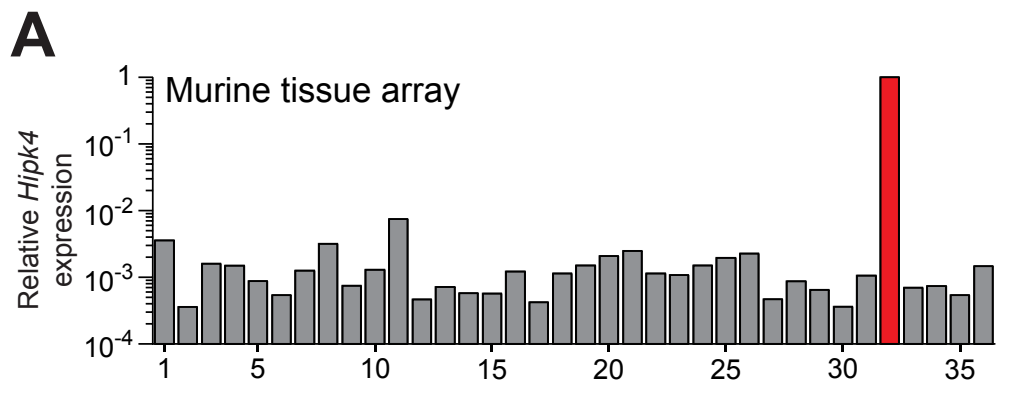

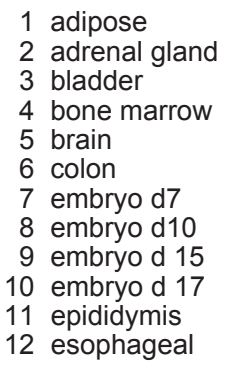

13 eye

14 heart

15 k dney

16 liver

17 lung

18 mammary, nonpreg.

19 mammary, preg. e10

20 mammary, preg. e15

21 mammary, invol. e1

22 mammary, lact. e3

23 muscle

24 ovary
25 placenta

26 prostate

27 skn

28 small intestine

29 spinal cord

30 spleen

31 stomach

32 testis

33 thymus

34 thyroid

35 trachea

36 uterus

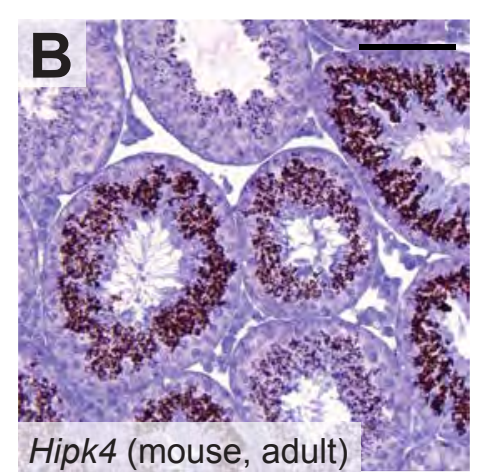

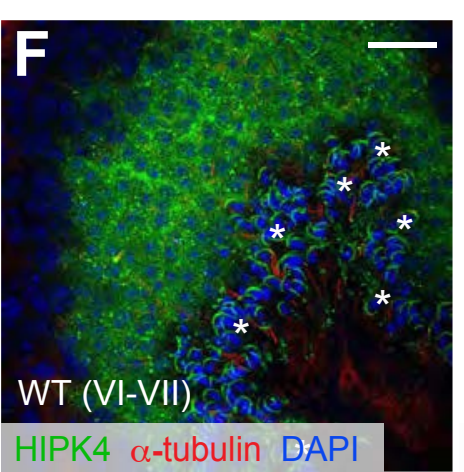
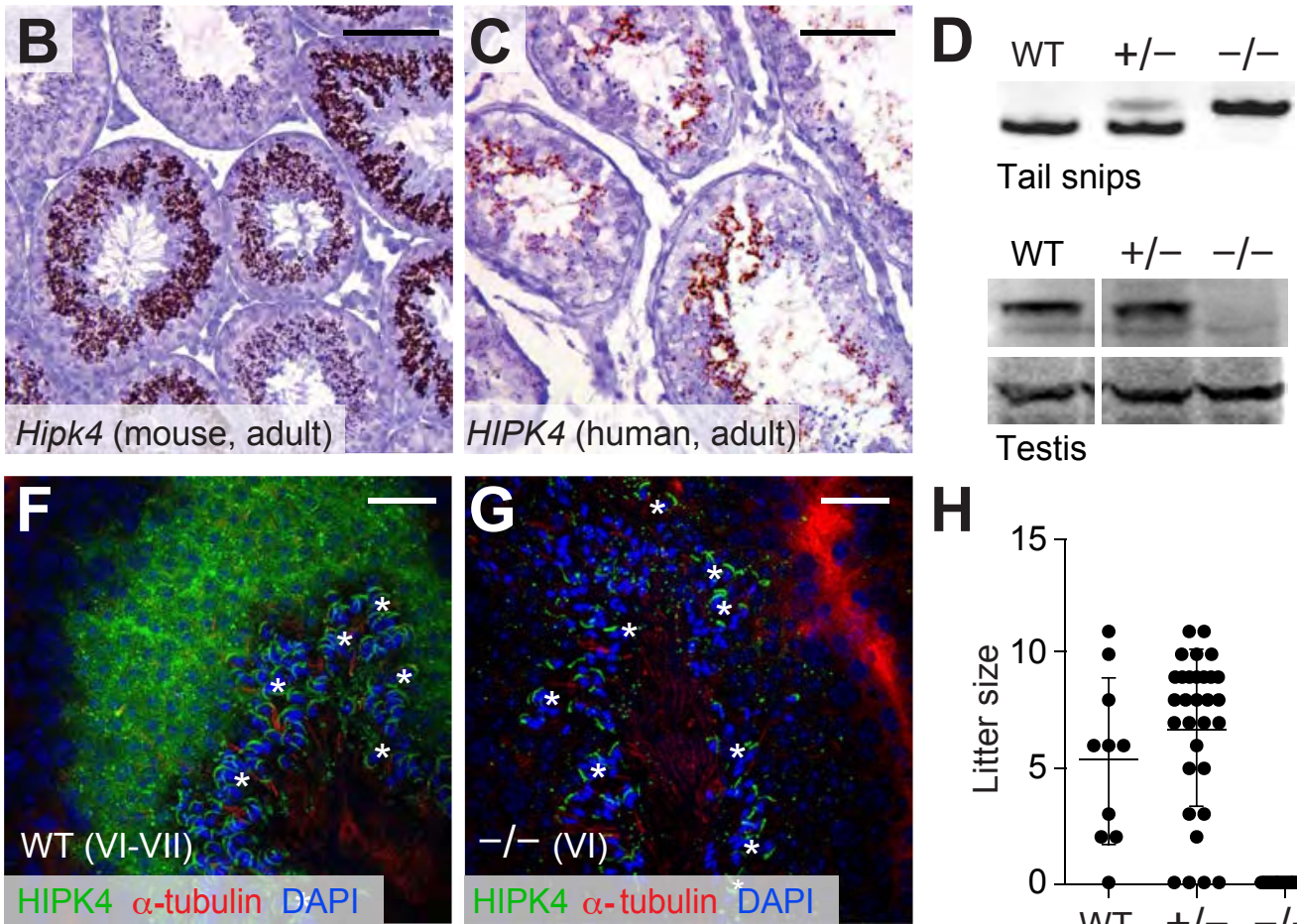

Tail snips
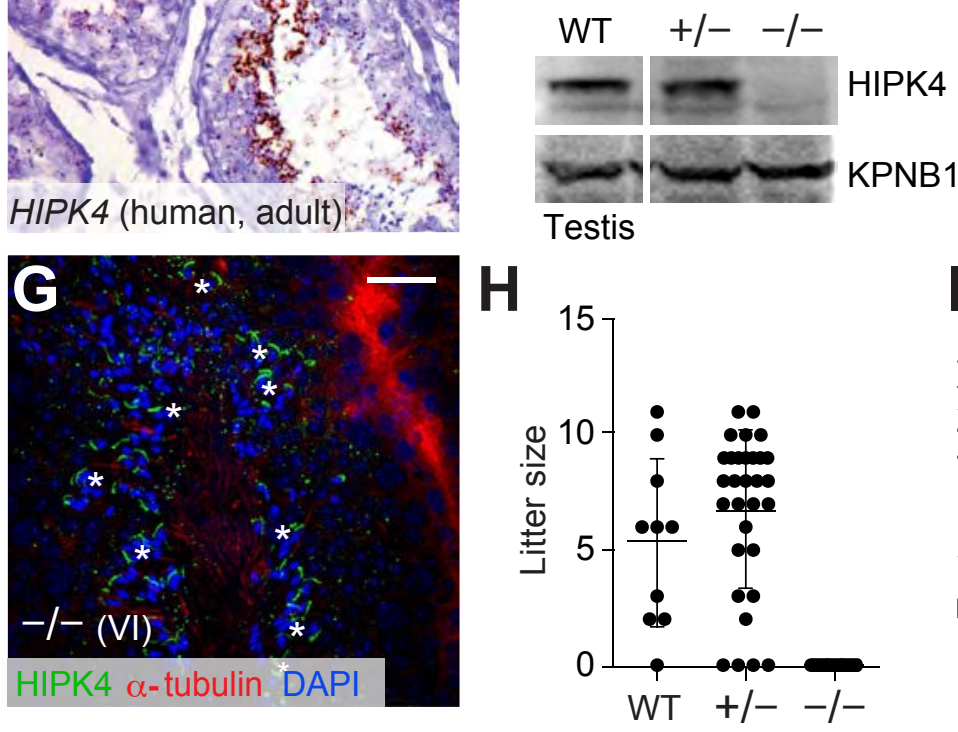

\begin{tabular}{ccc}
$\begin{array}{c}\text { Fertiliz tion method } \\
\text { (sperm type) }\end{array}$ & $\begin{array}{c}\text { Oocyte } \\
\text { number }\end{array}$ & $\begin{array}{c}\text { Fertiliz tion } \\
(2-\text {-cell) }\end{array}$ \\
\hline IVF (WT) & $*$ & $94 \%$ \\
IVF (-/-) & 69 & 0 \\
\hline ICSI (WT) & $*$ & $50-70 \%$ \\
ICSI (-/-) & 37 & $19(51 \%)$ \\
ICSI (-/-) & 37 & $32(86 \%)$
\end{tabular}

Embryogenesis (morula and blastocyst)

Pups
ND
ND
$10-15 \%$
ND
$4(11 \%)$

Figure 2. HIPK4 is expressed in spermatids and required for male fertility in mice. (A) Hipk4 expression in various murine tissues as determined by qPCR analysis of the Origene TissueScan ${ }^{\text {TM }}$ Mouse Normal cDNA array. Data are normaliz d to Gapdh. (B-C) Hipk4 expression in adult mouse (B) and human (C) testis sections as determined by in is tu hb ridia tion. (D) Validation of Hipk4 $\mathrm{k}$ ock ut by PCR of tail-derived genomic DNA and western blot analyses of whole testis lysates. Immunoblots are from the same membrane and exposure time. (E) Weights of WT, Hipk4 ${ }^{+/-}$, and Hipk4-l- males at 6-7 week of age. (F-G) HIPK4 protein expression in adult mouse seminiferous tubule sections (Stage VI-VII) as determined by immunofluorescence imaging. Asterisk indicate non-specific antibody binding (see Figure 2 figure supplement 2). (H) Number of live pups per litter resulting from crosses between 7-week old males and

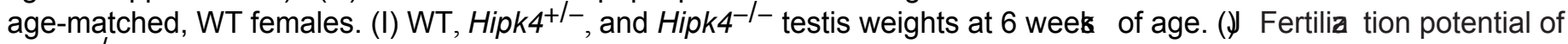
Hipk4 ${ }^{-/}$sperm using IVF and ICSI. ND = not determined. Experiments were conducted by the Stanford Transgenic, Knock ut, and Tumor Model Center, and the wild-type data represents the core facility's average results using C57BL/6NJ sperm. (K) Pups born via ICSI using Hipk4-/- sperm. Scale bars: B-C, $100 \mu \mathrm{m}$; F-G, $20 \mu \mathrm{m}$. Statistical analyses: error bars depicted in panels $\mathrm{E}, \mathrm{H}$, and I represent the average value \pm s.d. 
referred to as $\mathrm{Hipk}^{--}$) and confirmed that these mice fail to produce functional Hipk4 gene

153 products using in situ hybridization and western blot analysis (Figure 2D). Loss of HIPK4 had no

154 apparent effect on the animal viability or growth (Figure 2E). By immunostaining testis

155 cryosections from adult wild-type and Hipk $4^{--}$mice, we confirmed that HIPK4 protein is expressed

156 in round and early elongating spermatids (steps 3-8). The kinase is distributed throughout the

157 cytoplasm of these germ cells, mirroring its subcellular localization when overexpressed in

158 somatic cells (Figure 2F-G, Figure 2 - figure supplement 2) (van der Laden, et al., 2015).

159 Despite their grossly normal development and physiology, homozygous Hipk4 mutant males were unable to conceive, whereas heterozygote males sired normal litter sizes (Figure $1 \mathrm{H}-\mathrm{I}$ ). No significant differences in testis weight were observed across the Hipk4 genotypes (Figure 1J). Female Hipk4 ${ }^{--}$mice were physically indistinguishable from their wild-type and heterozygous

Hipk $^{--}$mice failed to fertilize wild-type oocytes under standard in vitro fertilization (IVF) could undergo uterine implantation to produce healthy pups (Figure 2J-K).

During our in vitro fertilization studies, it was apparent that the Hipk $4^{-1-}$ male mice had spermatogenesis defects consistent with OAT. In comparison to wild-type mice, homozygous

171 mutants produced sperm at low epididymal concentrations, and the germ cells had decreased

172 motility [both the total number of motile sperm and those with progressive motility as measured

173 by computer-assisted sperm analysis (CASA)] and abnormal morphology (Figure 3A-E, Figure 3

174 - figure supplement 1). Head defects included macrocephaly, microcephaly, and irregular shapes;

175 tail deformities included bent, coiled, crinkled, and shortened flagella. Hipk4 ${ }^{+/}$sperm also had

176 reduced epididymal concentrations and total motility, but their progressive motility and 177 morphology were normal. We observed that over $10 \%$ of the epididymal sperm isolated from 

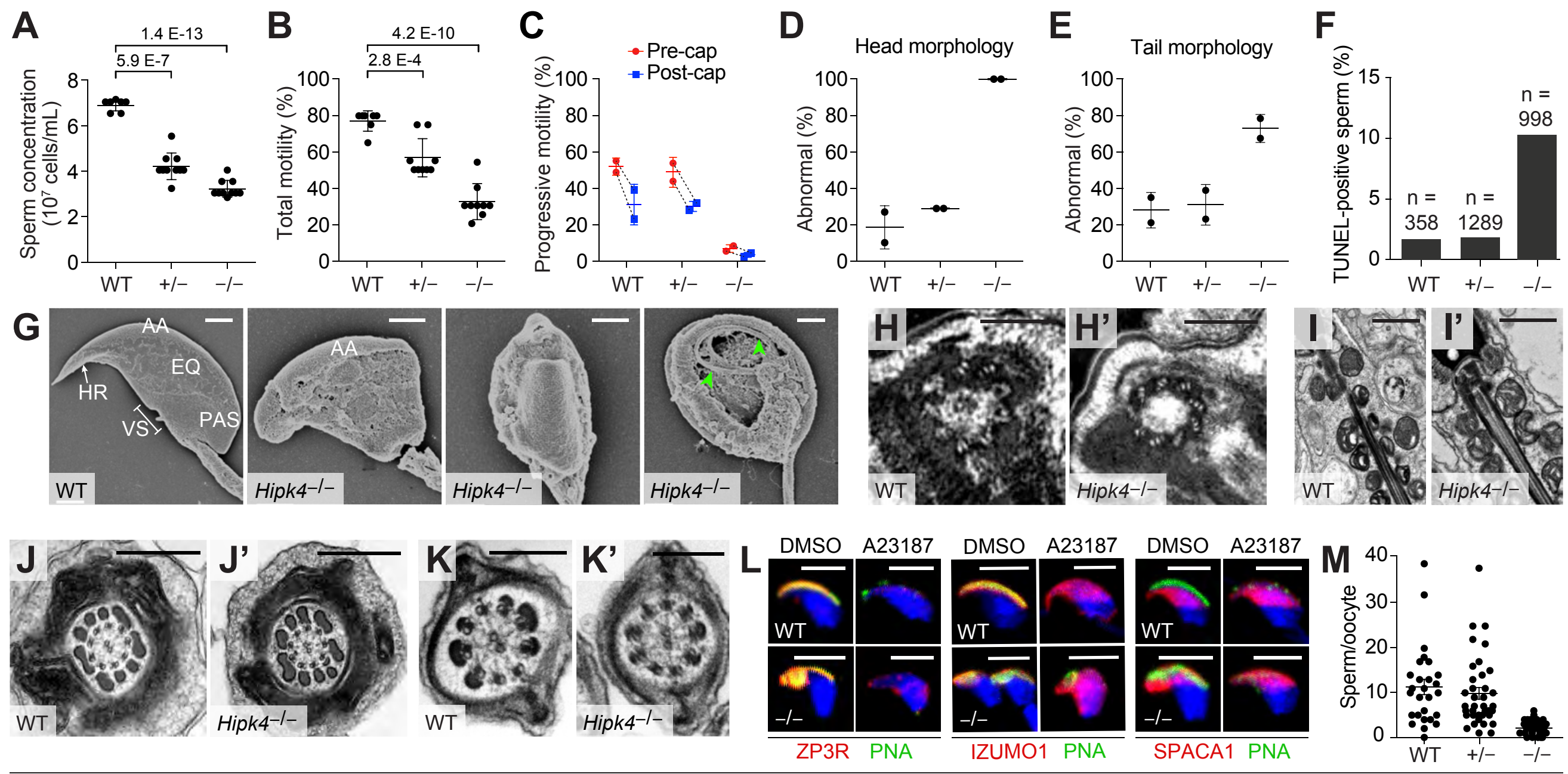

Figure 3. Hipk4 knockout mice exhibit oligoasthenoteratozoospermia. (A) Concentrations of epididymal sperm \pm s.d. $P$ values for the indicated statistical comparison are shown. (B) Percentage of epididymal sperm that were motile \pm s.d. $P$ values for the indicated statistical comparison are shown. (C) CASA measurements of progressive sperm motilty before and after capacitation \pm s.d. Data connected by the dashed lines are showing sperm motility from the same animal. (D, E) Percentage of sperm morphology with abnormal head or tail morphology \pm s.d. as assessed by phase contrast microscopy (see also, Figure 3 - Figure supplement 1 ). (F) Quantification of TUNEL-positive epididymal sperm with the designated genotypes $(n=$ total number of sperm analyE d). (G) SEM images of epididymal sperm. AA = anterior acrosome, EQ $=$ equatorial segment, PAS = postacrosomal segment, $\mathrm{VS}=$ ventral spur, HR = hook rim. Arrowheads point to the axoneme wrapped inside a demembranated sperm head. $\left(\mathrm{H}, \mathrm{H}^{\prime}\right)$ Centrioles at the basal body of step 15 spermatid axonemes. (I, I') Mitochondria along the midpiece of step 15 spermatids. ( $\left.\mathrm{J} J \mathrm{~J}\right)$ Cross section of step 15 spermatid flagella at the midpiece. (K, K') Cross section of step 15 spermatid flagella at their principal piece. (L) Acrosomal changes in capacitated sperm treated with a Ca ${ }^{2+}$ ionophore (A23187) as assessed by immunofluorescence and staining with FITC-labeled PNA. Nuclei were stained with DAPI. (M) Number of oocyte-bound sperm under standard IVF conditions after extensive washing \pm s.e.m. Scale bars: $\mathrm{G}, 2 \mu \mathrm{m} ; \mathrm{H}, 1 \mu \mathrm{m} ; \mathrm{I}, 1 \mu \mathrm{m} ; \mathrm{J} \quad 0.5 \mu \mathrm{m} ; \mathrm{K}, 0.2 \mu \mathrm{m} ; \mathrm{L}, 5 \mu \mathrm{m}$. 
$178 \mathrm{Hipk}^{--}$mice exhibited DNA fragmentation that could be detected by TUNEL (terminal 179 deoxynucleotidyl transferase dUTP nick end labeling) staining. In comparison, only $1.5 \%$ of wild180 type or Hipk4 ${ }^{+/-}$sperm were TUNEL-positive. Homozygous mutant sperm therefore may undergo 181 a higher rate of apoptosis, possibly accounting for their lower epididymal concentrations.

We further compared the head structures of wild-type and Hipk $4^{-1-}$ sperm by scanning electron microscopy (SEM; Figure 3G). All HIPK4-deficient sperm exhibited head morphologies 184 that deviated from the flat, crescent-shaped structures of their wild-type counterparts. Specific 185 defects included a disorganized anterior acrosome and the absence of a distinct equatorial 186 segment, post-acrosomal sheath, ventral spur, and sharp hook rim. In some sperm samples with demembranated head structures, we observed mislocalized axonemal components wrapped around the nucleus. We also used transmission electron microscopy (TEM) to analyze the tail structures of newly formed spermatozoa within the seminiferous tubule. HIPK4 loss did not appear to affect the basal body (Figure 3H-H'), axoneme (Figure 4I-K'), outer dense fibers (Figure 4I-I'), mitochondria (Figure 4J-J'), or fibrous sheath (Figure 4K-K') of these fully differentiated cells.

HIPK4-deficient sperm can undergo capacitation and the acrosome reaction in vitro

We next examined how the loss of HIPK4 affects two key aspects of sperm function: capacitation and the acrosome reaction. Sperm naturally undergo capacitation as they ascend the female reproductive tract and interact with the oviduct epithelium (Austin, 1951; Chang, 1951).

197 Changes in the glycoproteins, phospholipids, and cholesterol residing in the sperm plasma 198 membrane, $\mathrm{Ca}^{2+}$ influx, and increases in intracellular $\mathrm{pH}$ correlate with the activation of soluble 199 adenylate cyclase and protein kinase A (Abou-haila, et al., 2009; Lin, et al., 1996; O'Rand, 1982; 200 Stival, et al., 2016). Downstream protein tyrosine phosphorylation signaling events are initiated, 201 and sperm switch from progressive motility to a "hyperactivated" swimming motion (Alvau, et al., 202 2016; Naz, et al., 2004; Sepideh, et al., 2009). Capacitated sperm also become competent for the 203 acrosome reaction, during which the outer acrosomal membrane fuses with the overlying plasma 
membrane (Hirohashi, 2016; Kierszenbaum, 2000; Stival, et al., 2016). This process causes the release of digestive enzymes stored within the acrosome, and it exposes oocyte-binding receptors that are displayed on the inner acrosomal membrane, which spreads down over the equatorial region of the sperm head (Sebkova, et al., 2014; Sosnik, et al., 2009). Together, these steps ultimately promote sperm-oocyte engagement, fusion, and fertilization.

To determine whether HIPK4 is required for sperm capacitation, we isolated motile sperm

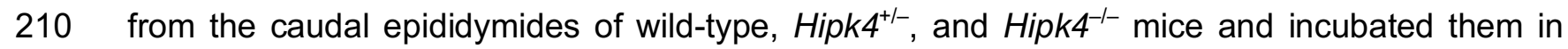
211 capacitation medium containing $\mathrm{Ca}^{2+}$, bicarbonate, and bovine serum albumin. We then assessed

212 the resulting levels of tyrosine phosphorylation ( $p$-Tyr) in soluble sperm lysates by western blot.

213 We observed no significant differences in p-Tyr between the three Hipk4 genotypes (Figure 3 -

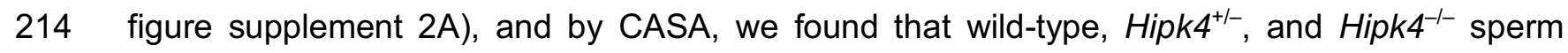
treated with capacitation medium undergo similar changes in progressive motility (Figure 3C). These results indicate that $H i p k 4^{--}$sperm are competent for capacitation in vitro.

To investigate the ability of HIPK4-deficient sperm to undergo acrosomal exocytosis, we

218 incubated capacitated sperm with the $\mathrm{Ca}^{2+}$ ionophore A23187. Sperm were then fixed, quickly 219 permeabilized, and labeled with fluorescein-conjugated peanut agglutinin (FITC-PNA), which 220 binds to the outer acrosome membrane and is lost upon exocytosis. After 1.5 hours of 221 capacitation, $86 \%$ of wild-type sperm had fully intact acrosomes, whereas only $63 \%$ of mutant 222 sperm retained acrosomes (due to either head malformations or spontaneous acrosome 223 exocytosis). As expected, A23187 treatment decreased the percentage of FITC-PNA positive 224 cells in both wild-type and Hipk $4^{--}$samples (to $22 \%$ and $45 \%$, respectively), although mutant 225 sperm responded less efficiently to this treatment.

227 proteins during A23187-induced exocytosis: ZP3R, IZUMO1, and SPACA1. ZP3R (also known 228 as sp56) is a zona pellucida-binding protein that appears on the sperm surface after capacitation, 229 and it is released when the outer acrosomal and plasma membranes fuse (Kim, K. S., et al., 
231 are membrane proteins required for head shaping and oocyte fusion that localize to distinct

232 acrosomal regions. IZUMO1 spreads from the anterior acrosomal cap to the equatorial segment

233 during the acrosome reaction (Inoue, et al., 2005; Sebkova, et al., 2014), and SPACA1 remains

234 localized to the equatorial region (Figure 3H) (Fujihara, et al., 2012). As assessed by

235 immunofluorescence microscopy, all three acrosomal factors exhibited HIPK4-independent

236 behaviors in response to $\mathrm{Ca}^{2+}$ ionophore exposure. Together, these findings suggest that HIPK4-

237 deficient sperm are capable of normal acrosomal changes, at least in response to calcium 238 signaling.

HIPK4-deficient sperm exhibit diminished oocyte binding

Since Hipk $4^{--}$sperm retain their ability to undergo capacitation and the acrosome reaction in vitro, we considered whether the head defects caused by HIPK4 loss might compromise spermoocyte interactions. Equivalent numbers of motile, capacitated sperm from wild-type, Hipk4 ${ }^{+/}$, or

244 Hipk $4^{-1-}$ males were incubated with cumulus-oocyte complexes (COCs) in human tubal fluid (HTF) 245 supplemented with $\mathrm{Ca}^{2+}$ and glutathione. The complexes were then washed repeatedly, fixed, 246 and oocyte-bound sperm were quantified by nuclear staining and confocal imaging (Figure 3l).

247 Consistent with the incompetence of $\mathrm{Hipk}^{-/-}$sperm for IVF, these cells bound less efficiently to 248 oocytes in comparison to their wild-type and heterozygous mutant counterparts. We further noted 249 that the COCs incubated with mutant sperm retained many cumulus cells, while all of the cumulus 250 cells of the COCs exposed to wild-type sperm were detached (Figure 3 - figure supplement 2).

251 Thus, HIPK4-deficient sperm lack structural and/or molecular features that are required for 252 maximally productive sperm-oocyte interactions. 
Loss of HIPK4 function disrupts the acrosome-acroplaxome complex

To gain insights into the spermatogenic defects caused by loss of HIPK4, we compared periodic acid-Schiff (PAS)-stained testis sections obtained from adult wild-type and $H i p k 4^{-1-}$ mice. Hipk $4^{-/-}$testes contained malformed elongating spermatids, which failed to properly extend by step 12 (Figure 4A-A', Figure 4 - figure supplement 1A-B). In addition, stage IX and X tubules from mutant mice contained spermatozoa that were still attached to Sertoli cells. Since these mature germ cells are normally released into the seminiferous lumen by stage VIII, HIPK4deficient spermatozoa appear to have delayed spermiation, which may also contribute to the

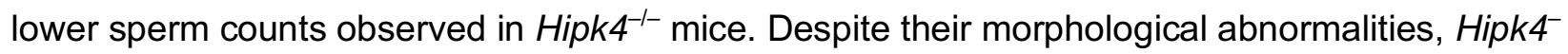
I- spermatozoa released to the epididymis progressed normally to the cauda (Figure 4B-B', Figure 4 - figure supplement 1C-D).

Next, we analyzed spermatid structures in greater detail by TEM imaging of testis sections. Although HIPK4 protein expression peaks in step 5-7 spermatids, all Hipk $4^{--}$round spermatids appeared normal by TEM (Figure 4C-C', Figure 4 - figure supplement 2A). Spermatids begin to elongate at step 8, and most of these cells appeared normal in Hipk $4^{-1-}$ testes. However, some HIPK4-null step 8 spermatids contained highly amorphous, fragmented acrosomal vesicles and/or

271 detached acrosomal granules, which were coincident with structural abnormalities to the anterior

272 nuclear pole (Figure 4 - figure supplement $2 A$ ). Aberrant head structures became universally 273 apparent in step 9-10 Hipk4 ${ }^{-/}$spermatids (Figure 4C-C'). The acrosome-acroplaxome marginal 274 ring was no longer juxtaposed to the perinuclear ring of the manchette, significantly widening the 275 groove belt and deforming the underlying nuclear lamina (Figure 4D-D'). In some cases, 276 enlargement of the groove belt was accompanied by detachment of the acrosome, severe anterior 277 head deformities, and retention of spermatid cytoplasm (Figure 4 - figure supplement 2B). We 278 also observed small electron-dense areas at the posterior boundary of the acroplaxome that have 279 not been previously defined. These electron-dense structures appeared to interact with the 

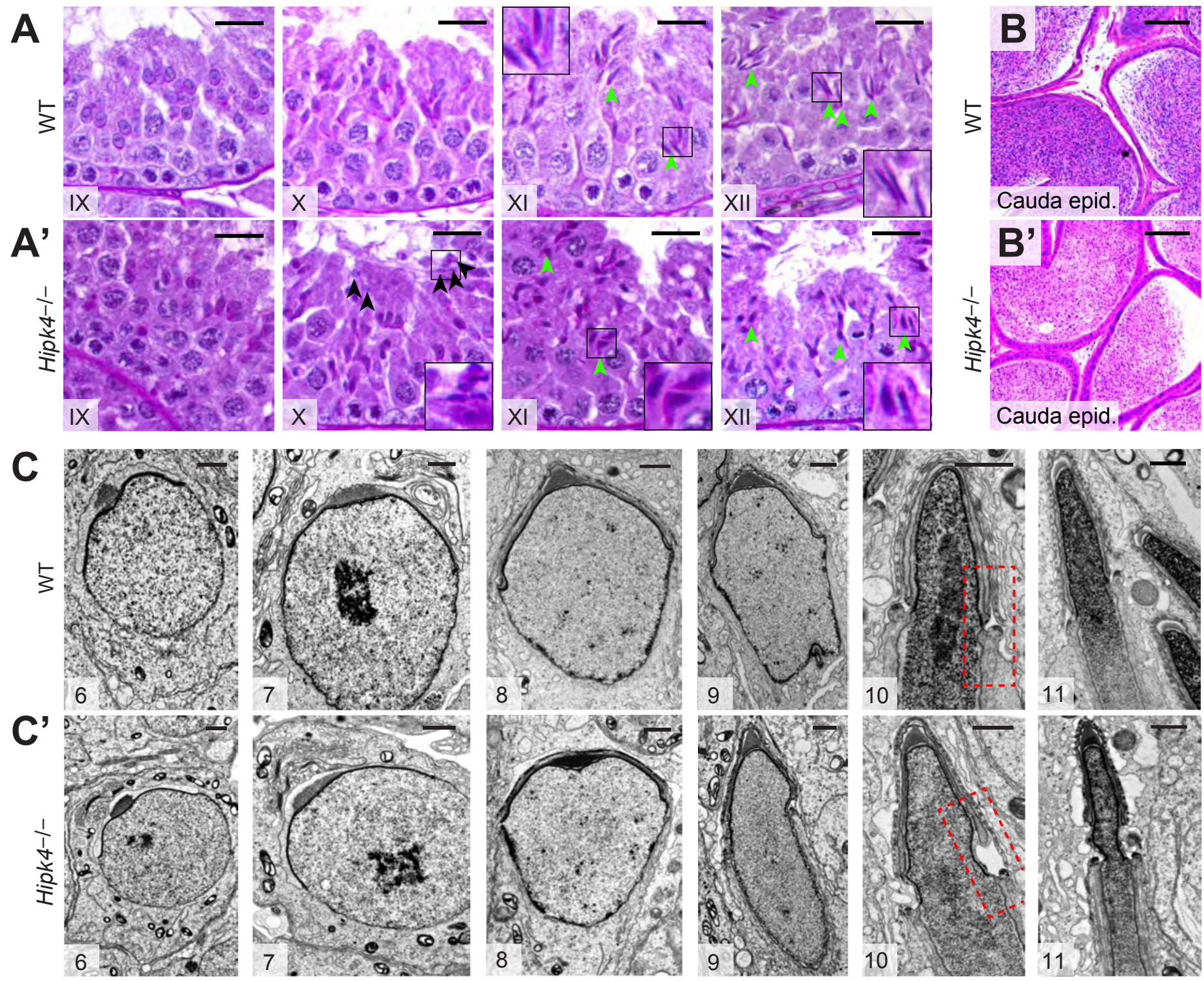

D
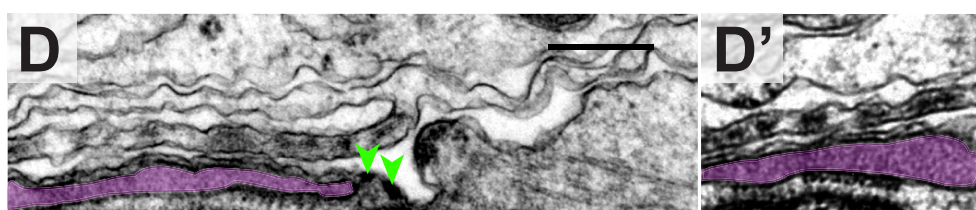

\section{$A$}

Hipk $4^{-1-}$

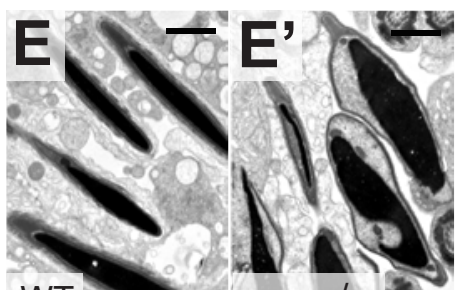

WT

WT

Hipk4-1-

Figure 4. HIPK4 regulates the acrosome-acroplaxome complex. (A,A') PAS-stained sections of seminiferous tubules from WT or Hipk4-I- mice at various stages of spermatogenesis. Black arrowheads point to testicular spermatoø a that are retained in a stage $X$ Hipk $4^{-/}$tubule. Green arrowheads point to the heads of elongating spermatids. (B, B') H\&E-stained sections of caudal epididymides from WT or Hipk4-l- mice. (C-E) TEM images. (C, C') Step 6-11 spermatids from WT or Hipk4-l- mice. Areas outlined by the red dashed boxes are shown at higher magnification in D-D'. (D, D') Magnified view of the groove belt region of step 10 spermatids. Green arrowheads point to electron densities in the acroplaxome that are normally associated with the posterior edge of the acrosome (highlighted in purple). Blue arrowheads point to filaments link ng the manchette to the nuclear envelope. (E, E') TEM images of condensed spermatids in WT and Hipk $^{-l-}$ testis sections. Scale bars: A-B, $20 \mu \mathrm{m} ; \mathrm{C}, 1 \mu \mathrm{m} ; \mathrm{D}, 0.2 \mu \mathrm{m} ; \mathrm{E}, 2 \mu \mathrm{m}$. 
acrosome in wild-type spermatids, but they were disorganized and uncoupled from this organelle in HIPK4-null cells (Figure 4D-D', Figure 4 - figure supplement 2C).

In contrast to the acroplaxome-acrosome defects, other anterior head structures appeared to form properly in HIPK4-deficient spermatids. For example, the anterior nuclear membrane

284 (nuclear dense lamina) is putatively anchored to the acroplaxome via the inner membrane protein

285 DPY19L2, while the outer acrosomal membrane is connected to the cytoskeleton via a LINC 286 complex composed of SUN1 and nesprin3. These membrane-cytoskeleton linkages had 287 comparable subcellular distributions in wild-type and Hipk $4^{-/-}$spermatids, as assessed by 288 immunofluorescence imaging of isolated germ cells (Figure $4-$ figure supplement 3A-B). TEM 289 analyses similarly revealed no overt defects within the manchette, including the perinuclear ring 290 and the conical array of filaments that scaffold the posterior nuclear pole (Figures 4C-C', 4D-D').

291 We further characterized manchette formation and degradation by immunostaining the microtubule end-binding protein EB3 in testis cryosections and isolated germ cells. Testis sections from Hipk $4^{--}$mice exhibited wild-type-like manchette dynamics (Figure 4 - figure supplement 3C). However, elongating Hipk $4^{-/-}$spermatids isolated from dissociated testis tissues occasionally exhibited abnormally shaped manchettes (Figure 4 - figure supplement 3D), suggesting that HIPK4 may also regulate certain aspects of this microtubule- and F-actin-scaffolded structure.

HIPK4 does not primarily act through transcriptional regulation

Given the delay between the onset of HIPK4 expression in wild-type round spermatids and the emergence of HIPK4-dependent morphological phenotypes, we investigated whether HIPK4 301 signaling could regulate the acrosome-acroplaxome complex through transcriptional 302 mechanisms. Other HIPK family members and related kinases (e.g., DYRKs) localize to the 303 nucleus and have established roles in transcriptional regulation (Di Vona, et al., 2015; Rinaldo, et 304 al., 2008), and HIPK4 can phosphorylate p53 in vitro (Arai, et al., 2007). We therefore used 305 oligonucleotide microarrays with full-genome coverage to profile transcriptional differences 


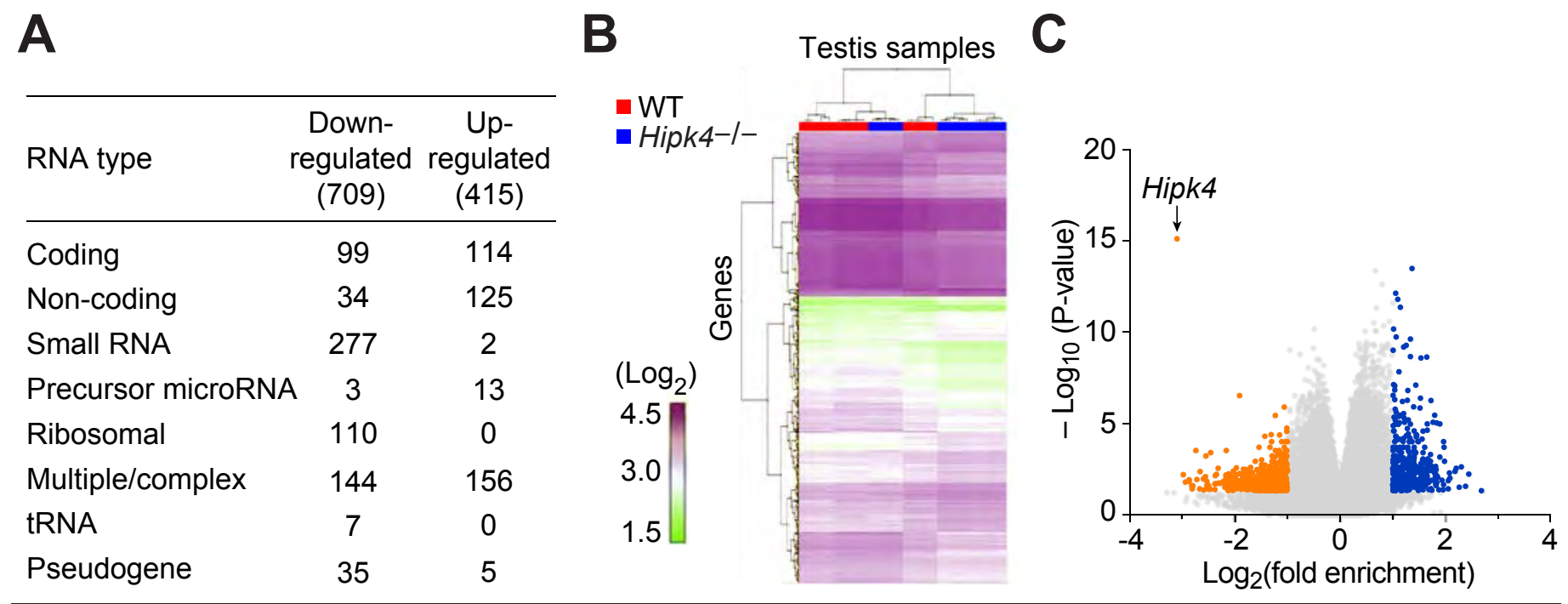

Figure 5. Wild-type and Hipk4 mutant testes have similar transcriptomes. (A) Summary of the types of RNA that were increased (up-regulated) or decreased (down-regulated) in Hipk4 $\mathrm{k}$ ock ut testes compared to wild-type, as measured by quadruplicate microarray analysis of three testis samples. (B) Histogram of normaliz d signal intensities ( $\left.\log _{2}\right)$ of gene expression levels in individual microarrarys. Color scheme was arbitrarily assigned. (C) Volcano plot depicting the transcriptional differences between Hipk $4 \mathrm{k}$ ock ut and wild-type testes. RNA species that exhibited a $>2$-fold change in abundance and had a $P$-value $<0.05$ are shown in orange (down-regulated) or blue (up-regulated). 
between adult testes isolated from wild-type and Hipk $4^{--}$mice. Through this approach, we identified 415 genes that were upregulated $\geq 2$-fold in Hipk $4^{-1-}$ testes compared to wild-type tissue and 709 genes that were downregulated $\geq 2$-fold (Figure 5A and Figure 5 - Source data 1). Hierarchical clustering of the data from biological replicates revealed overlapping groupings of wild-type and Hipk $4^{--}$samples (Figure 5B), indicating that the transcriptional differences between the genotypes are modest. Hipk4 itself exhibited the largest change in transcript abundance between genotypes (Figure 5C). We also noted that loss of HIPK4 did not significantly alter the mRNA levels of transcription factors that are unique to stage 5-7 seminiferous tubules (Green, et al., 2018). Based on these microarray data, we surmise that HIPK4 does not primarily act through transcriptional regulation.

HIPK4 overexpression remodels F-actin in cultured somatic cells spindle-like morphologies, whereas cells expressing HIPK4 became either spherical or polygonal within two days after infection. The polygonal HIPK4-overexpressing cells were multinucleate, 324 likely due to cytokinesis failure (Figure 6A-C). Coincident with these changes in cell shape, we observed a striking loss of F-actin-containing stress fibers in the HIPK4-overexpressing cells

326 (Figure 6D-E). The overall ratio of globular actin (G-actin) to F-actin did not increase with Hipk4 327 transduction (Figure 6F), suggesting that this kinase induces F-actin remodeling rather than 328 filament disassembly into monomeric subunits. HIPK4 overexpression did not overtly alter the 329 microtubule cytoskeleton in these cells (Figure 6G-H).

330 We then compared the phosphoproteomes of NIH-3T3 cells transduced with wild-type 331 HIPK4, the K40S variant, or a second inactive mutant that is incapable of autophosphorylation- 
bioRxiv preprint doi: https://doi.org/10.1101/703637; this version posted July 16, 2019. The copyright holder for this preprint (which was not certified by peer review) is the author/funder, who has granted bioRxiv a license to display the preprint in perpetuity. It is made available under
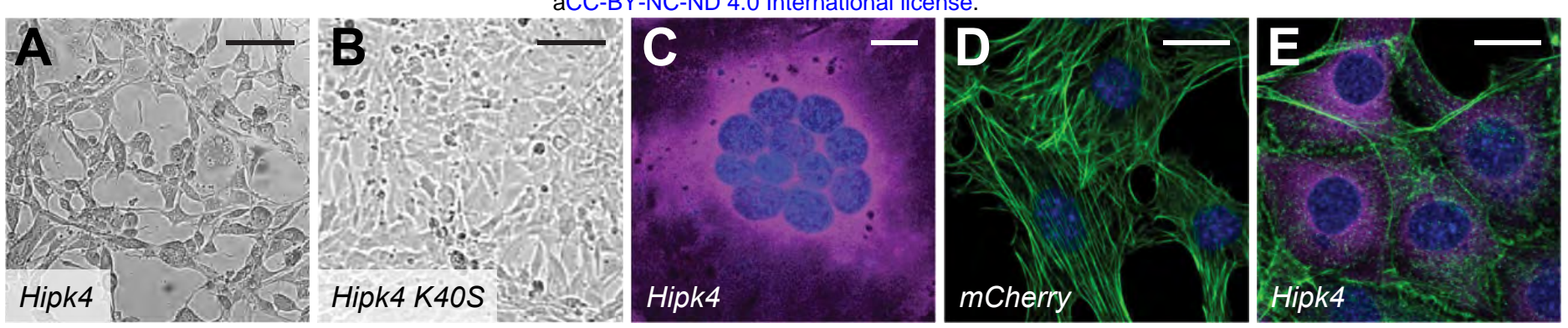

FLAG (HIPK4) DAPI Phalloidin
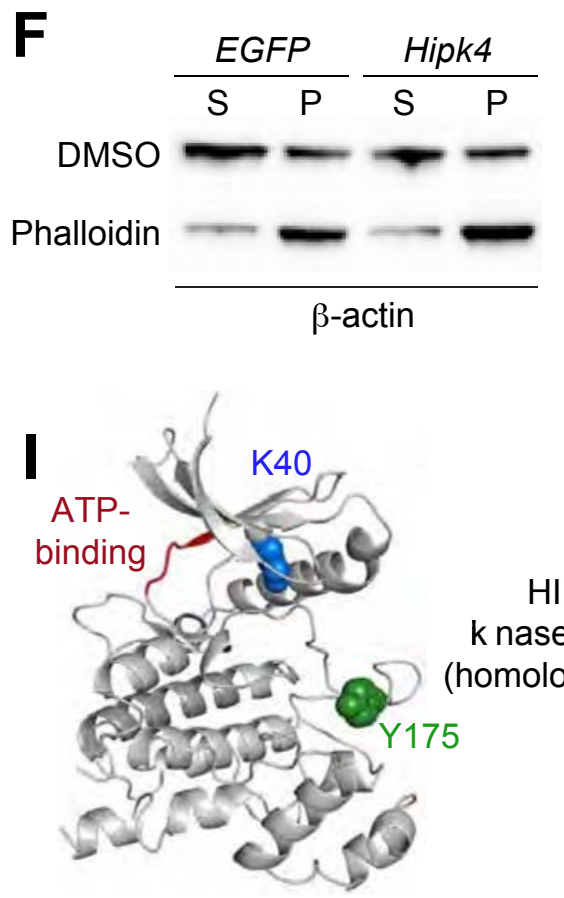

J
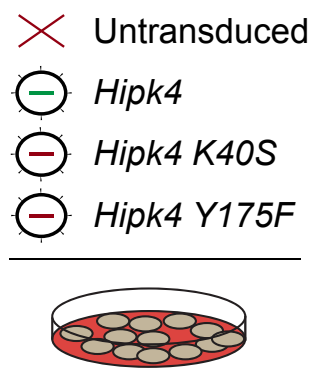

Retrovirally transduced NIH-3T3 cells
Lysates digested (Trypsin/LysC)
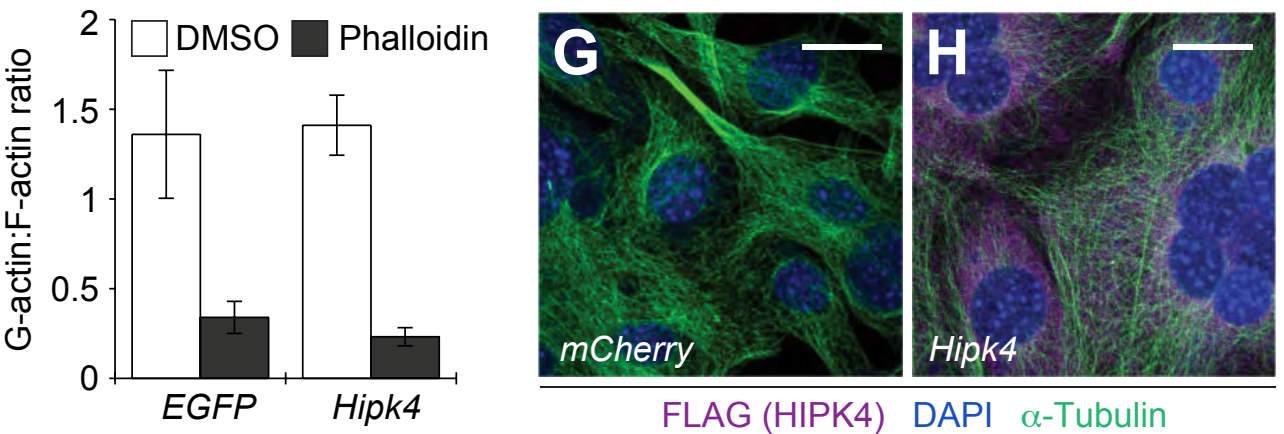

$\mathrm{K}$

HIPK4

$\mathrm{k}$ nase domain (homology model)

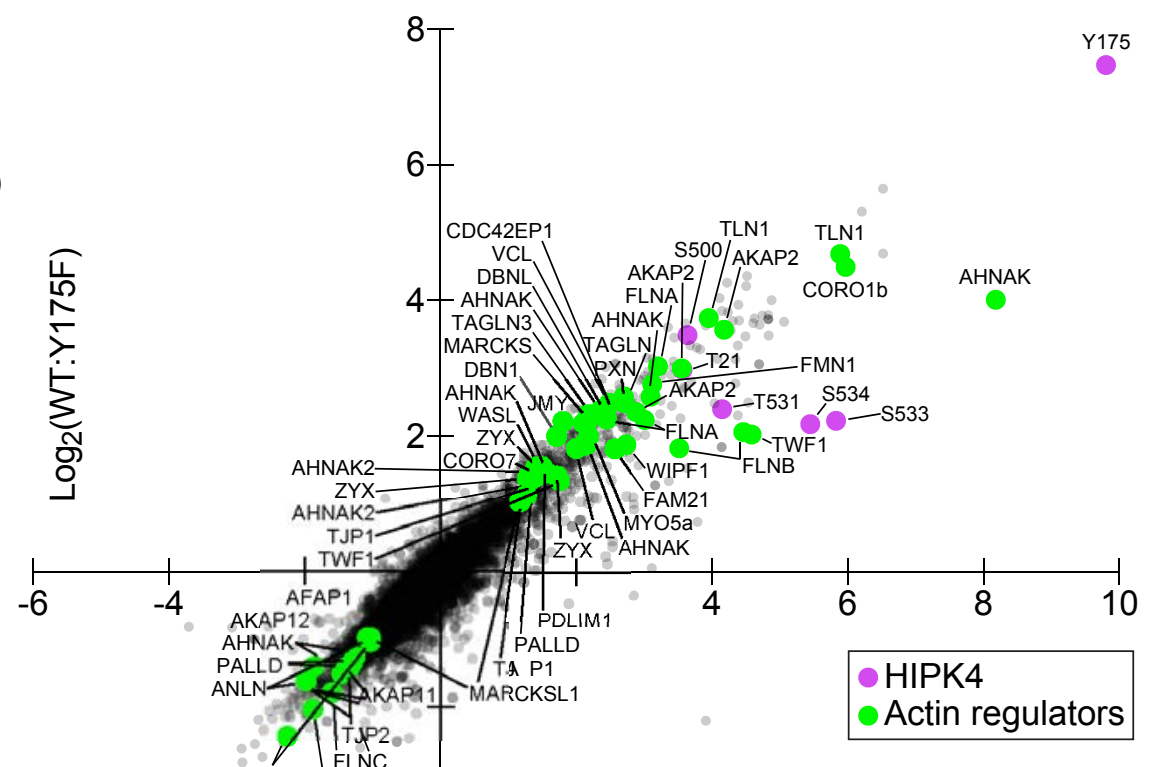

Phosphopeptide enrichment

(Ti-IMAC \& $\mathrm{TiO}_{2}$ )<smiles>[VH]</smiles>

TMT-labeling

High $\mathrm{pH}$, reverse-phase chromatography $\downarrow$ LC-MS/MS 10 FLNA MACF1

$$
\begin{aligned}
& -4- \\
& -6-
\end{aligned}
$$

$\log _{2}(\mathrm{WT}: \mathrm{K} 40 \mathrm{~S})$

Figure 6. Hipk4 overexpression remodels the actin cytoskeleton in cultured cells. (A-B) Brightfield images of NIH-3T3 cells retrovirally transduced with FLAG-tagged Hipk4 or k nase-dead Hipk4 K4OS. (C) HIPK4-expressing NIH-3T3 cell with multiple nuclei. (D-E) Phalloidin and anti-FLAG staining of NIH-3T3 cells transduced with mCherry or FLAG-tagged Hipk4. (F) G- and F-actin levels in NIH-3T3 cells transduced with EGFP or FLAG-tagged Hipk4 and then treated with DMSO or phalloidin after lysis. The western blot shows the soluble (S; G-actin) and pelleted (P; F-actin) pools of actin after ultracentrifugation, and the graph depicts the average G-actin:F-actin ratios of quadruplicate samples \pm s.e.m. $(\mathrm{G}-\mathrm{H}) \alpha$-Tubulin and FLAG immunostaining of NIH-3T3 cells transduced with mCherry or FLAG-tagged Hipk4. (I) Homology model of the HIPK4 $\mathrm{k}$ nase domain using the DYRK1A structure as a template (PDB ID: 3ANQ). The ATP-binding site is colored red, and residues that are essential for catalytic activity are depicted as blue or green space-filling models. (ل Work flow used to characterie the HIPK4-dependent phosphoproteome in NIH-3T3 cells. (K) Scatter plot of 6,941 phosphosites identified by LC-MS/MS, graphed according to their relative levels in NIH-3T3 cells overexpressing wild-type or catalytically inactive HIPK4. Selected HIPK4-regulated phosphosites in actin-modulating proteins are shown in green and phosphosites in HIPK4 are shown in purple. Scale bars: A-B, $100 \mu \mathrm{m} ; \mathrm{C}-\mathrm{E}$ and G-H, $20 \mu \mathrm{m}$. 
332 dependent activation (Y175F) (van der Laden, et al., 2015) (Figure 6I-J). Through phosphopeptide 333 enrichment, isobaric labeling, and quantitative mass spectrometry, we identified 6,941

334 phosphosites; 303 of which increased in abundance by $\geq 2$-fold upon HIPK4 overexpression (in 335 comparison to either inactive mutant) (Figure 6K and Figure 6 - Source data 1). Consistent with 336 the effects of HIPK4 overexpression on F-actin dynamics, several of these phosphosites reside 337 in known actin-interacting proteins. For example, we identified HIPK4-dependent phosphosites in 338 talin 1 (TLN1), AHNAK nucleoprotein, coronin 1B (CORO1B), A-kinase anchor protein 2 (AKAP2), 339 formin 1 (FMN1), vinculin (VCL), MARCKS, paxillin (PXN), WASH family members (WASL, 340 WIPF1, and FAM21), zyxin (ZYX), unconventional myosin 5a (MYO5a), filamins (FLNA and 341 FLNB), and transgelins (TAGLN and TAGLN3).

Because HIPK4 overexpression altered stress fiber dynamics in cultured cells, we hypothesized that HIPK4 may similarly modulate F-actin-related functions in developing sperm. In particular, a role for HIPK4 in regulating acroplaxome structure and/or function could explain the head defects observed in Hipk4 ${ }^{--}$spermatids. We first used fluorescently labeled phalloidin to visualize F-actin structures in wild-type and $\mathrm{Hipk}^{-/-}$testis sections; however, since the phalloidin staining predominantly labeled the F-actin hoops of Sertoli cells, it was difficult to discern differences between the samples (Figure 7A). We consequently used the phalloidin probe to analyze spermatids isolated from dissociated testes, which enabled us to specifically image F-

352 actin in the acroplaxome. Although there was no discernable difference in acroplaxome F-actin in 353 round-to-early elongating spermatids isolated from wild-type and Hipk $4^{--}$testes (Figure 7B), wild354 type but not $\mathrm{Hipk}^{-/-}$germ cells maintained this F-actin-based plate at later stages of spermatid 355 differentiation (e.g., condensed spermatids). To further compare F-actin-related structures in wild356 type and $\mathrm{Hipk}^{-/-}$spermatids, we assessed the localization of actin-capping proteins CAPZA3 357 and CAPZB3 by immunofluorescence microscopy (Figure 7C-D). Consistent with our phalloidin 
A

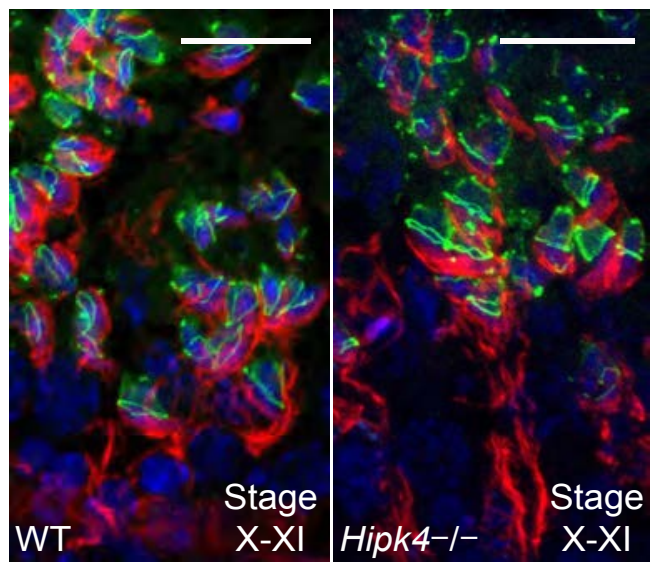

Phalloidin EB3 DAPI

C
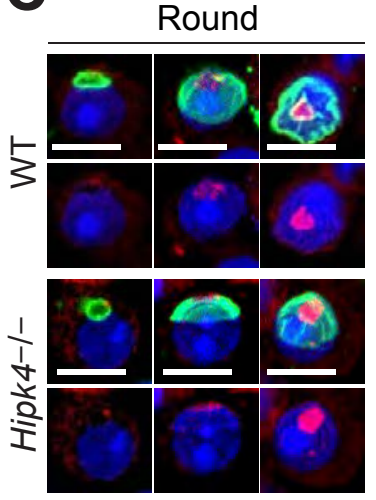

CAPZA3 PNA Hoechst 33342

E

WT

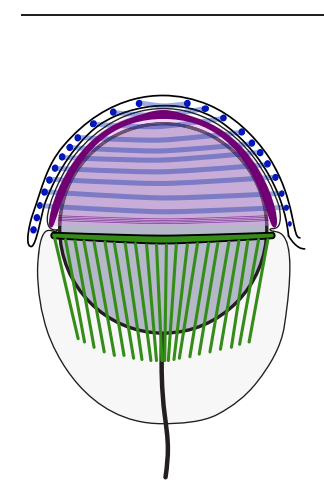

External forces from Sertoli cell F-actin hoops

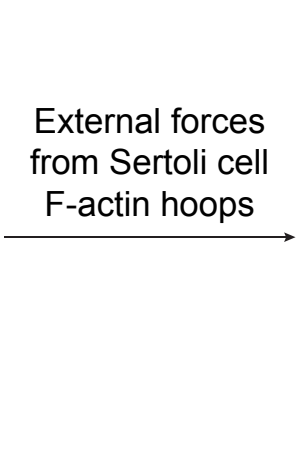

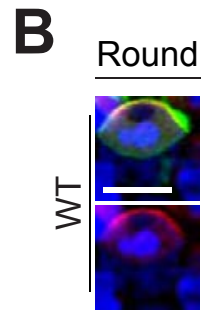
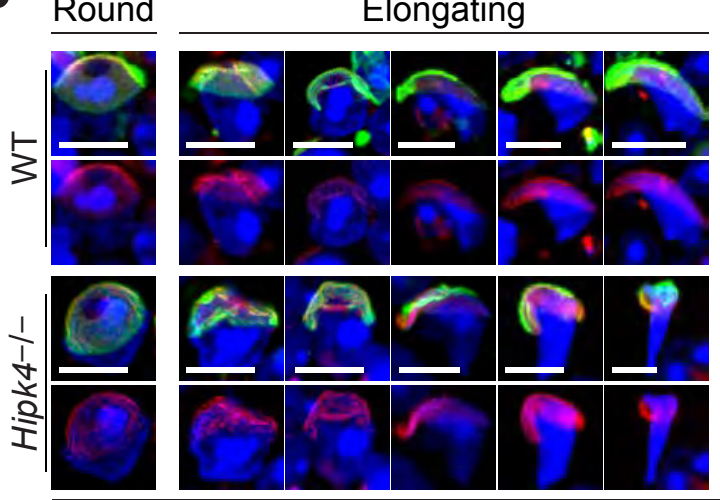

Condensing/Condensed
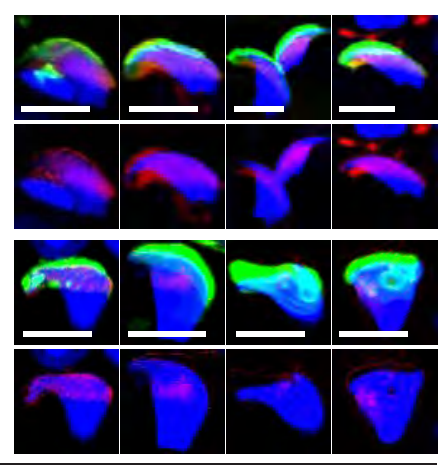

Phalloidin PNA Hoechst 33342

Condensing/ Condensed

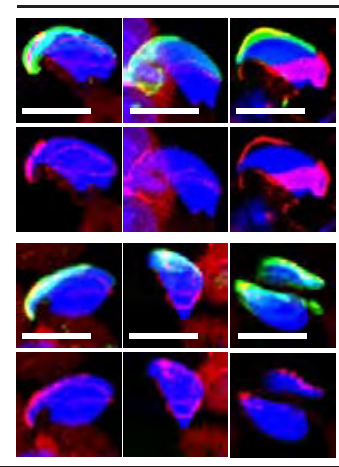

D

Dound
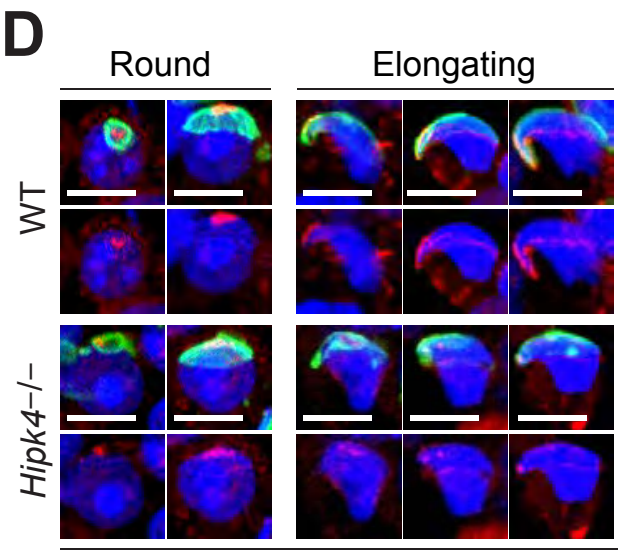

Condensing/ Condensed

CAPZB3 PNA Hoechst 33342

Hipk4-/-
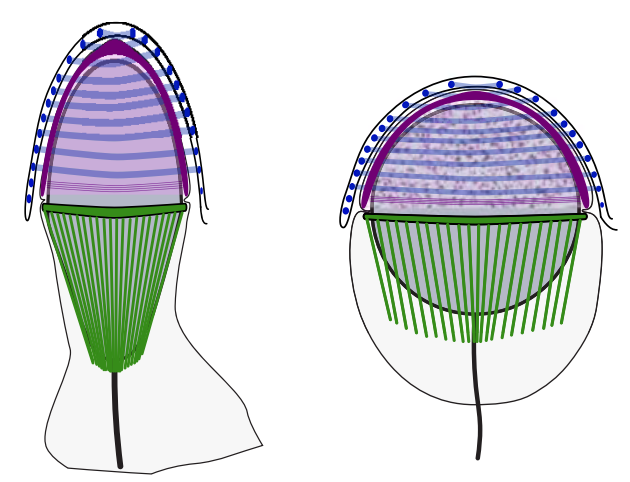

External forces from Sertoli cell F-actin hoops

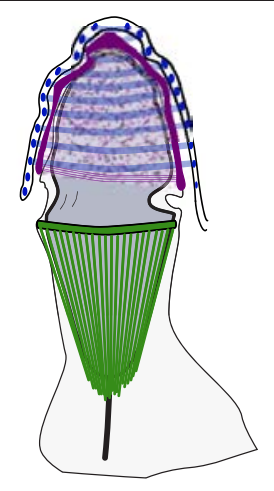

Figure 7. Loss of HIPK4 function alters F-actin dynamics in the acroplaxome. (A) Cryosectioned seminiferous tubules (stage X/XI) stained with Alexafluor-647-phalloidin and Alexafluor-488-anti-EB3 antibody to label basal and apical Sertoli cell ectoplasmic specializ tions. Nuclei were stained with DAPI. (B-D) Enzm atically dissociated elongating spermatids stained with FITC-PNA and Alexafluor-647-phalloidin (B), anti-CAPZA3 (C), or anti-CAPZB3 (D). Nuclei were stained with Hoeschst 33342. (E) A model for HIPK4 function during spermiogenesis. Scale bars: A, $20 \mu \mathrm{m}$; B-D, $2 \mu \mathrm{m}$. 
staining results, elongating wild-type and Hipk $4^{-1-}$ spermatids exhibited similar expression levels and subcellular distributions of CAPZA3 and CAPZB3, but these actin regulators were selectively diminished in condensing Hipk4 ${ }^{-/-}$spermatids.

\section{DISCUSSION}

HIPK4 is a dual-specificity kinase that is predominantly expressed in differentiating spermatids, and it has been previously reported that HIPK4 deficiency in mice can alter sperm morphology and number. Our studies reveal both the cell biological basis and reproductive consequences of these spermatogenic phenotypes. Our findings establish HIPK4 as an important regulator of the acrosome-acroplaxome complex during spermiogenesis. Hipk $4^{-1-}$ spermatids form abnormal acroplaxome structures, and this F-actin- and keratin-containing plate becomes uncoupled from the Golgi-derived acrosome, most overtly at the groove belt in these mutant cells. HIPK4 function is also required to coordinate acrosome-acroplaxome and manchette dynamics

371 during spermatid elongation, as evidenced by the grossly enlarged groove belt in elongating 372 Hipk $4^{-1-}$ spermatids. The resulting spermatozoa have misshapen heads, and a large fraction 373 exhibit irregular tail morphologies. Hipk $4^{-1-}$ males have lower concentrations of epididymal sperm, 374 and these mutant germ cells have lower motility than their wild-type counterparts. Together, these 375 spermatogenic defects closely mirror those observed in men with severe OAT syndrome, and like 376 these clinical cases, HIPK4-deficient male mice are sterile.

Of these phenotypes, the abnormal head structures of HIPK4-deficient sperm are likely a 378 dominant cause of sterility. Sperm produced by heterozygous Hipk4 mutant males have lower 379 than normal epididymal concentrations of sperm and reduced total motility, but the fertility of these 380 animals is comparable to that of wild-type mice. Only homozygous Hipk4 mutant germ cells exhibit 381 head defects that become increasingly overt during spermiogenesis. Consistent with diminished 382 head functions, Hipk $4^{--}$sperm are also incompetent for IVF and have reduced binding and 383 penetration of COCs. This diminished fertility could reflect structural defects that physically 
abrogate sperm-egg interactions and/or concomitant perturbations that disrupt molecular processes within the head. The ability of $H i p k 4^{-/-}$sperm to undergo capacitation and the acrosome reaction in vitro suggests that key intracellular biochemical pathways remain intact. Nevertheless, we cannot rule out the possibility that HIPK4 is required for these processes in vivo. The reduced motility of Hipk4 ${ }^{-/-}$sperm could also affect fertility within the female reproductive tract.

Our investigations reveal potential mechanisms by which HIPK4 could regulate the acrosome-acroplaxome complex. HIPK1-3 have been shown to directly phosphorylate homeodomain transcription factors (Kim, Y. H., et al., 1998; Rinaldo, et al., 2008), and the structurally related dual-specificity tyrosine-regulated kinase 1a (DYRK1A) targets various transcriptional activators and repressors (Di Vona, et al., 2015; Litovchick, et al., 2011; Mao, et al., 2002; Woods, et al., 2001; Yang, et al., 2001). Although it has been reported that HIPK4 can phosphorylate p53 in vitro (Arai, et al., 2007; He, et al., 2010), it is unlikely that HIPK4 acts primarily through transcriptional control. In contrast to other HIPK family members, HIPK4 lacks the homeobox-interacting domain and a nuclear localization sequence, and accordingly it localizes to the cytoplasm rather than the nucleus (van der Laden, et al., 2015). Moreover, our genome-wide microarray analyses reveal that loss of HIPK4 function does not lead to major transcriptional changes within the testis. These observations suggest that HIPK4 regulates the acrosome-acroplaxome complex through more direct biochemical mechanisms, and our cell biological studies strongly implicate HIPK4 in F-actin remodeling. HIPK4 expression in cultured

403 somatic cells promotes the formation of branched F-actin structures and alters the 404 phosphorylation state of multiple actin-crosslinking proteins. HIPK4 could similarly modulate F405 actin networks within the acroplaxome. Consistent with this model, HIPK4 deficiency disrupts the 406 levels of F-actin and actin-capping proteins localized to the acroplaxome marginal ring in 407 condensing spermatids (CAPZA3 and CAPZB3), perhaps due to cytoskeletal dysregulation at 408 earlier stages. We further note that transcripts encoding actin cytoskeleton components are 409 among the few genes that are expressed in a HIPK4-dependent manner within the testis (Figure 
5 - Source data 1). For example, the actin-related protein Actr6 and the actin-capping protein

411 Capza2 were downregulated in $\mathrm{Hipk}^{-1-}$ testis tissues, and upregulated genes includes those that

412 encode actin-membrane crosslinkers (TIn2, Flnb, and Ank2), actin-based motors (Myo5b and

413 Myo10), and components of the acrosome matrix (Zp3r and Zan). We speculate that these

414 transcriptional changes reflect cellular responses to cytoskeletal defects caused by HIPK4

415 deficiency.

416 Based on our findings, we propose a model in which HIPK4 promotes F-actin remodeling in

417 the acroplaxome, modulating its biophysical properties and interactions with the acrosome

418 (Figure 7E). We hypothesize that loss of HIPK4 function alters acroplaxome stability and

419 dynamics, rendering this cytoskeletal plate less able to distribute external forces applied by the

420 Sertoli cell F-actin hoops. As a result, the spermatid nucleus fails to elongate properly, and the

421 head structure becomes grossly misshapen. HIPK4 deficiency also uncouples the acrosome-

422 acroplaxome complex at the groove belt, leading to severe anterior malformations that may

423 disrupt the expulsion of excess cytoplasm and other aspects of spermatid differentiation. It is likely

424 that germ cells with such significant defects are less competent for spermiation and less viable

425 after their release into seminiferous tubule lumen, which could account for the lower

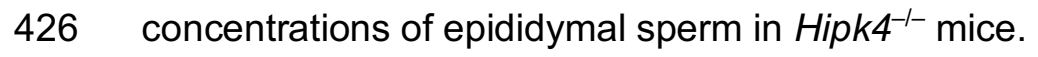

HIPK4 joins the small collection of protein kinases that are known to be enriched or

428 exclusively expressed in haploid male germ cells and required for spermatogenesis. For example,

429 the kinases CSNK2A2, SSTK, and CAMKIV function in the spermatid nucleus and promote the

430 histone-to-protamine transition (Escalier, et al., 2003; Spiridonov, et al., 2005; Wu, et al., 2000).

431 TSSK1 and 2 are testis-specific kinases that regulate the chromatoid body, centrioles, and the

432 developing sperm flagellum (Jha, et al., 2013; Kueng, et al., 1997; Shang, P., et al., 2010; Xu, et

433 al., 2008), and TSSK4 and TSSK5 localize to the flagellum and control sperm motility (Wang, et

434 al., 2016; Wang, et al., 2015). The only kinase known to be required for spermatid head shaping

435 is TR-KIT, a splice variant of c-KIT that regulates manchette formation and later has a role in 
436 oocyte activation upon fertilization. A truncated, testis-specific version of FER, FERT,

437 phosphorylates cortactin in the acroplaxome (Keshet, et al., 1990; Kierszenbaum, et al., 2008),

438 but male mice lacking FERT are still fertile (Craig, et al., 2001). To the best of our knowledge,

439 HIPK4 is the first kinase known to be essential for acrosome-acroplaxome function and male

440 fertility.

441 Given the druggable nature of kinases, HIPK4 is also a new potential target for male 442 contraception. Small-molecule HIPK4-specific antagonists could recapitulate the male sterility

443 caused by disrupting the Hipk4 gene in mice. Such treatments would reduce male fertility without

444 interfering with the hypothalamus-pituitary-gonadal axis, damaging the testis or germline, or

445 introducing genetic abnormalities to offspring if fertilization is achieved. Since HIPK4 expression

446 is restricted to the final stages of sperm development, HIPK4 inhibitors also would induce sterility

447 more quickly than drugs that perturb earlier steps in spermatogenesis. Contraceptive reversibility

448 would be equally rapid. Moreover, HIPK4 antagonists could have minimal non-reproductive

449 effects, as Hipk4 $4^{-1-}$ mice appear to have otherwise normal physiology. Further investigations of

450 HIPK4 therefore could not only elucidate the mechanisms that drive spermatid differentiation but

451 also address a longstanding unmet need in reproductive medicine. 


\section{MATERIALS AND METHODS}

\section{Ethics statement}

All animal studies were conducted in compliance with the Stanford University Institutional Animal Care and Use Committee under Protocol 29999. Vertebrate research at the Stanford

457 University School of Medicine is supervised by the Department of Comparative Medicine's

458 Veterinary Service Center. Stanford University animal facilities meet federal, state, and local 459 guidelines for laboratory animal care and are accredited by the Association for the Assessment 460 and Accreditation of Laboratory Animal Care International. De-identified human testis sections 461 were obtained from Stanford University in compliance with protocol IRB-32801.

Animal use

Hipk $^{+/ t m 1 b}$ breeding pairs (C57BL/6NJ background, Stock No. 025579) and wild-type (C57BL/6NJ, Stock No. 005304) mice were purchased from The Jackson Laboratory. Mice used for this study were weaned at 19-22 dpp and genotyped using Platinum Taq DNA Polymerase

467 (Invitrogen) and the following primers: wild-type forward 5'-CCTTTGGCCTTATACATGCAC-3', wild-type reverse 5'-CAGGTGTCAGGTCTGGCTCT-3', mutant forward 5'-CGGTCGCTACCATT ACCAGT-3', mutant reverse 5'-ACCTTGAGATGACCCTCCTG-3'.

Tissue distribution of Hipk4

TaqMan primers (Applied Biosystems) for Hipk4 (Mm01156517_g1) were used to probe the

473 Origene TissueScan ${ }^{\mathrm{TM}}$ Mouse Normal cDNA Array according to the manufacturer's protocols.

474 Gene expression levels were normalized to GAPDH (Mm99999915_g1).

476 In situ hybridization analysis

477 Whole testes were dissected and immediately fixed in freshly prepared modified Davidson's 478 fixative (30\% formaldehyde, $15 \%$ ethanol, $5 \%$ glacial acetic acid, $50 \%$ distilled water) for 
16-20 hours and then washed and stored in 70\% ethanol until further use. For in situ hybridization analyses, the fixed tissues were paraffin-embedded, cut into $10-\mu \mathrm{m}$ sections, and mounted on slides. To detect Hipk4 transcripts, we used the RNAscope ${ }^{\circledR} 2.5$ HD Detection Kit (Advanced Cell Diagnostics) with Hipk4 probes (Mm-Hipk4, 428071), following the manufacturer's protocol for formalin-fixed, paraffin-embedded (FFPE) sections. Hybridization probes for Ppib (BA-Mm-Ppib1ZZ, 313911) and dapB (BA-DapB-1ZZ, 310043) were used as positive and negative controls, respectively.

Assessment of fecundity

To test fertility by mating, we paired $\geq 7$-week-old males (wild-type, Hipk4 ${ }^{+-}$, and Hipk $4^{-1-}$ ) with age-matched, wild-type females for 18-21 days, and the number of live-born pups for each pairing was recorded. Male and female mice were paired in this manner for 2-4 rounds (6-12 weeks).

IVF and ICSI procedures were performed at the Transgenic, Knockout, Tumor Model Center at Stanford University. For IVF studies, C57BL/6NJ females were superovulated by injection with 494 PMSG (ProSpec, HOR-272, 5U, 61-63 hours prior to oocyte-harvesting) and hCG (ProSpec, 495 HOR-250, 5U, 48 hours after PMSG injection). On the day of the experiment, epididymal sperm 496 were isolated using a "swim-out" method in TYH medium (120 mM NaCl, $5 \mathrm{mM} \mathrm{KCl,} 2.5 \mathrm{mM}$ $497 \mathrm{MgSO}_{4}, 1.0 \mathrm{mM} \mathrm{KH}_{2} \mathrm{PO}_{4}, 25 \mathrm{mM} \mathrm{NaHCO} 3,2.5 \mathrm{mM} \mathrm{CaCl}_{2}, 1 \mathrm{mM}$ sodium pyruvate, $1.0 \mathrm{mg} / \mathrm{mL}$ 498 glucose, $1.0 \mathrm{mg} / \mathrm{mL}$ methyl- $\beta$-cyclodextrin, and $1.0 \mathrm{mg} / \mathrm{mL}$ polyvinylalcohol; $\mathrm{pH}$ 7.2) and 499 transferred to TYH medium containing bovine serum albumin (BSA; final concentration of $5005 \mu \mathrm{g} / \mathrm{mL}$ ). Cumulus-oocyte complexes (COCs) were then harvested from the oviducts of these 501 superovulated females in M2 medium with HEPES buffer (Sigma, M7167), and transferred 502 through three $50 \mu \mathrm{L}$ drops of HTF medium (Millipore, MR-070-D) containing $0.25 \mathrm{mM}$ reduced 503 glutathione under mineral oil, pre-equilibrated to $37{ }^{\circ} \mathrm{C}, 5 \% \mathrm{CO}_{2}$. After 1 hour of capacitation, 504 motile sperm $\left(\sim 5.0 \times 10^{5}\right)$ were added to the drop of HTF medium containing COCs and incubated 
505 for 4 hours at $37^{\circ} \mathrm{C}$ and $5 \% \mathrm{CO}_{2}$. Sperm-oocyte complexes were then washed four times in M2

506 medium and incubated in $30 \mu \mathrm{L}$ KSOM medium (Millipore, MR-101-D) at $37{ }^{\circ} \mathrm{C}, 5 \% \mathrm{CO}_{2}$ under

507 mineral oil. The number of two-cell, morula, and blastocyst-stage embryos were then counted

508 over the next 72 hours.

509 ICSI experiments were performed as previously described (Yoshida, et al., 2007). Briefly,

510 motile sperm were harvested from the epididymis, and sperm heads were injected into the

511 cytoplasm of CD1 oocytes using a piezo-actuated micromanipulator. The injected embryos were

512 cultured in $\mathrm{KSOM}$ medium at $37^{\circ} \mathrm{C}$. After 24 hours, live two-cell embryos were either cultured

513 until the blastocyst stage or implanted into the oviducts of pseudo-pregnant female mice.

Antibodies

The following primary antibodies were used for western blotting and immunofluorescence

517 imaging: anti-HIPK4 (FabGennix International, rabbit pAb generated against the peptide sequence PAGSKSDSNFSNLIRLSQVSPED, lot 1651.Pb1.AP); anti-KPNB1 (H-300) (Santa Cruz Biotechnology, sc-11367, rabbit pAb); anti-phosphotyrosine (Upstate/Millipore, 4G10 ${ }^{\circledR}$ Platinum, 05-1050, lot 2723728, rabbit pAb); anti-ZP3R/mouse sp56 (7C5) (QED Bioscience, 55101, lot 051614-120816, mouse mAb); anti-IZUMO1 (125) (Abcam, ab211626, lot GR279965-4, rat mAb); anti-SPACA1 (Abcam, ab191843, lot GR312512-3, rabbit pAb); anti-FLAG ${ }^{\circledR}$ (M2, Sigma, F3165, 523 mouse mAb); anti-alpha tubulin (3H3085) (Santa Cruz Biotechnology, sc-69970, lot G0109, rat 524 mAb); anti-CAPZA3 (Progen, GP-SH4, lot 804091, guinea pig pAb); anti-CAPZB3 (Progen, GP525 SH5, lot 804081, guinea pig pAb); anti-DPY19L2 (gift from Christophe Arnoult, rabbit pAb), anti526 SUN1 (gift from Manfred Alsheimer, guinea pig pAb); anti-nesprin3 (gift from Arnoud Sonnenberg, 527 rabbit pAb); Alexa Fluor ${ }^{\mathrm{TM}}$ 488-conjugated anti-EB3 (EPR11421-B) (Abcam, ab203264, lot 528 GR227133-1, rabbit pAb).

529 Secondary antibodies included HRP-conjugated sheep anti-mouse IgG (GE Healthcare, 530 NA931V, lot 9682503), HRP-conjugated donkey anti-rabbit IgG (GE Healthcare, NA934V, lot 
9780721), Alexa Fluor ${ }^{\mathrm{TM}}$ 594-conjugated anti-guinea pig (Invitrogen, A11076, lot 1924784), Alexa

532 Fluor $^{\mathrm{TM}}$ Plus 647-conjugated goat anti-mouse IgG (Invitrogen, A32728, lot UB275580), Alexa

533 Fluor $^{\mathrm{TM}}$ 647-conjugated goat anti-rat IgG (Invitrogen, A21247, lot 37177A), and Alexa Fluor ${ }^{\mathrm{TM}}$ Plus

534 647-conjugated goat anti-rabbit lgG (Invitrogen, A32733, lot TL272452).

Western blot analysis

To detect HIPK4 protein, testis lysates were prepared by sonication of $\sim 100$-mg pieces of freshly removed whole testis in $1.0 \mathrm{~mL}$ ice-cold RIPA buffer containing protease inhibitors (cOmplete ${ }^{T M}$, EDTA-free Protease Inhibitor Cocktail Tablets, Roche, 4693159001) and phosphatase inhibitors (PhosSTOP ${ }^{T M}$, Roche, 4906837001). Equivalent amounts (12 $\left.\mu \mathrm{g}\right)$ of total protein per sample were diluted with $6 x$ Laemmli sample buffer, boiled for 5 minutes, and loaded on a $3-8 \%$ tris-acetate gel (Bio-Rad) for SDS-PAGE. Proteins were transferred to a PVDF

543 membrane and immunoblotted with the following primary antibodies: anti-HIPK4 [0.55 $\mu \mathrm{g} / \mathrm{mL}$,

544 1:1000 dilution in phosphate buffered saline (PBS) containing $0.1 \%$ Tween 20 and $4 \%$ BSA] or

545 anti-KPNB1 (1:1000). Chemiluminescence detection was conducted with HRP-conjugated

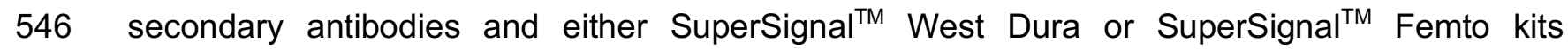
547 (Pierce/Thermo Fisher Scientific, 34076 or 34095, respectively).

Assessment of sperm quality before being cut open with fine scissors in $200 \mu \mathrm{L}$ HTF medium equilibrated at $37{ }^{\circ} \mathrm{C}$ and $5 \%$

$552 \mathrm{CO}_{2}$. The sperm suspension $(10 \mu \mathrm{L})$ was counted and assessed for total motility at $100 \mathrm{x}$ magnification using Leja slides and a microscope equipped with a reticle. Sperm quality 554 parameters of males (15-17 weeks old) were also assessed by the Mouse Biology Program at 555 UC-Davis using the following procedures. Morphology was assessed visually, and CASA was 
1000x magnification to determine the percentage with abnormal head sizes and shapes

558 (macrocephalous, microcephalous, tapered, triangular, olive, pin, banana, amorphous, collapsed,

559 abnormal hook, irregularly shaped, etc.) or abnormal midpieces or tails (bent, coiled, short, thin,

560 crinkles, irregularly shaped, etc.). Sperm were not included in the morphology assessment if they

561 were: (1) aggregated; (2) had a back or abdomen view of the head; or (3) were decapitated. At

562 least 100 sperm in 5 or more fields of view were evaluated for each experimental condition.

Assessment of sperm maturation and function

To assess capacitation by western blot, motile sperm were collected in $2.5 \mathrm{~mL}$ of TYH medium (BSA-free, $\mathrm{pH} 7.4$, and $\mathrm{CO}_{2}$-equilibrated) from cauda epididymides using the "swim out" method at $37{ }^{\circ} \mathrm{C}$ and $5 \% \mathrm{CO}_{2}$. The sperm suspension was then centrifuged at $600 \mathrm{~g}$, and all but $0.4 \mathrm{~mL}$ of the supernatant was removed to achieve a final concentration of $\sim 40 \times 10^{6}$ cells $/ \mathrm{mL}$. In fresh polystyrene tubes, $200 \mu \mathrm{L}$ of the sperm solution was added to $1.0 \mathrm{~mL}$ TYH medium containing BSA $(10 \mathrm{mg} / \mathrm{mL})$, and the suspension was incubated at $37^{\circ} \mathrm{C}$ for 1.5 hours. The sperm

571 were then centrifuged at $13,000 \mathrm{~g}$ for 1 minute, washed with PBS containing phosphatase

572 inhibitors, and pelleted once more. After the addition of $1 \mathrm{x}$ Laemmli sample buffer without 573 reducing agent $(25 \mu \mathrm{L})$, and the samples were boiled for 5 minutes and centrifuged at 13,000 $\mathrm{g}$.

574 The supernatants were collected and $2 \mu \mathrm{L}$ of $\beta$-mercaptoethanol was added to each sample, 575 which were then boiled again for 1 minute. Following SDS-PAGE, proteins were transferred to a 576 PVDF membrane and immunoblotted with an anti-phosphotyrosine antibody (1:1000 dilution, 577 Upstate/Millipore) for chemiluminescence detection.

578 To perform in vitro acrosome reactions, sperm were collected and capacitated as described 579 above. Following 1.5 hours of capacitation, the $\mathrm{Ca}^{2+}$ ionophore $\mathrm{A} 23187$ (hemicalcium salt; Sigma, $580 \mathrm{C} 275 ; 1000 \mathrm{x}$ stock dissolved in ethanol) was added to the suspensions to achieve final 581 concentrations of $10 \mu \mathrm{M}$. After incubation for 1 hour at $37{ }^{\circ} \mathrm{C}$ and $5 \% \mathrm{CO}_{2}$, and the cells were 
583 following steps were then performed in microcentrifuge tubes, and the cells were pelleted at 600

$584 \mathrm{~g}$ and washed with PBS between each step. Sperm were fixed by adding $2.0 \mathrm{~mL} 4 \%$ 585 paraformaldehyde in PBS for 15 minutes and permeabilizing with $0.3 \%$ Triton-X100 in PBS for 5

586 minutes. For experiments measuring acrosome exocytosis, the sperm were then incubated for 30

587 minutes in PBS containing 2\% BSA, 0.01\% Triton X-100, and fluorescein-conjugated peanut

588 agglutinin (FITC-PNA, $10 \mu \mathrm{g} / \mathrm{mL}$, Sigma, L7381, lot 046M4030V) and $10 \mu \mathrm{g} / \mathrm{mL}$ Hoechst 33342

589 nuclear stain. After washing the cells with PBS, they were mounted on microslides with

590 Vectashield Vibrance medium (Vector Labs, H-1700, ZE1011). For immunolabeling experiments,

591 the cells were then divided into separate tubes, blocked for 1 hour with PBS containing $2 \%$ BSA

592 and $0.01 \%$ Triton $\mathrm{X}-100$, incubated for 1 hour at room temperature with primary antibodies (10

$593 \mu \mathrm{g} / \mathrm{mL}$ final concentration in blocking buffer), and then washed once with blocking buffer. The

594 samples were then incubated with fluorescently labeled secondary antibodies and $10 \mu \mathrm{g} / \mathrm{mL}$

595 FITC-PNA in blocking buffer for 30 minutes. The sperm were washed once more, transferred to

596 microscope slides, mounted with Prolong Gold medium with DAPI, and then imaged on a Zeiss

597 LSM 700 confocal microscope equipped with a 63x oil-immersion objective. ImageJ software

$598(\mathrm{NIH})$ was used to create maximum-intensity Z-stack projections, and Photoshop CS6 (Adobe)

599 was used to crop images and adjust fluorescence intensity levels.

600 To assay for oocyte binding in vitro, we followed the same general protocol described for

601 IVF experiments. After 3 hours of sperm-COC incubation and five washing steps, we transferred

602 the complexes in a small volume into a $50-\mu \mathrm{L}$ drop of PBS containing $4 \%$ paraformaldehyde on a

603 microscope slide. After 30 minutes, excess liquid was carefully removed with a Kimwipe, and the

604 slides were mounted with Prolong Gold medium with DAPI. Fluorescence and DIC imaging on a

605 Leica DMi8 microscope at 200x magnification were used to assess the number of sperm bound 606 to oocytes. 
TUNEL assays

To measure DNA double strand breaks by terminal deoxynucleotidyl transferase-mediated

611 dUTP nick end labeling (TUNEL), we used the In Situ Cell Death Detection Kit (Sigma-Aldrich,

612 11684795910), following the manufacturer's protocol (ver. 17) for cell suspensions. Fluorescence

613 imaging was performed using a Zeiss LSM 700 confocal microscope equipped with a $63 x$ oil-

614 immersion objective. ImageJ software (NIH) was used to create maximum-intensity Z-stack

615 projections and TUNEL-positive cells were manually counted $(n=>357)$.

Histology of testis and epididymis

Histological staining (PAS and H\&E) was performed on formalin-fixed, paraffin-embedded testis and epididymis sections $(10 \mu \mathrm{m})$. To obtain these sections, the tissues were removed from 12- to 15-week-old males, immediately fixed in modified Davidson's fixative for 16 hours, and stored in $70 \%$ ethanol until paraffin-embedding, sectioning, and mounting on microscope slides. The slides then were heated for 1 hour at $50{ }^{\circ} \mathrm{C}$, washed with xylenes, re-hydrated, and stained with periodic acid solution (Sigma, 3951) and Schiff's reagent (Sigma, 3952016) and/or

624 counterstained with modified Harris hematoxylin solution (Sigma, HHS32) and Eosin Y (Sigma, 625 E4009).

\section{Electron microscopy}

For scanning electron microscopy, epididymal sperm were collected in TYH medium by the

629 "swim-out" method at $37^{\circ} \mathrm{C}$, pelleted at $200 \mathrm{~g}$, resuspended in $0.1 \mathrm{M}$ sodium cacodylate buffer $630(\mathrm{pH} 7.4)$ containing $2 \%$ paraformaldehyde and $4 \%$ glutaraldehyde, and transferred to individual 631 wells of a 24 -well plate containing poly-D-lysine-coated 12-mm coverslips. The samples were 632 allowed to fix overnight at $4{ }^{\circ} \mathrm{C}$ and then post-stained with $1 \%$ aqueous osmium tetroxide (EMS, 633 19100) for 1 hour. $\mathrm{OsO}_{4}$-treated samples were rinsed in ultrafiltered water three times and 634 gradually dehydrated in increasing concentrations of ethanol $(50 \%, 70 \%, 90 \%, 2 \times 100 \%$; 
63515 minutes each). Each coverslip was then dried at the critical point with liquid $\mathrm{CO}_{2}$ using a

636 Tousimis Autosamdriß-815A system and 15-minute purge time. Dried samples were sputter-

637 coated $(100 \AA, A u / P d)$ before imaging with a Zeiss Sigma FE-SEM using In-Lens and lateral 638 Secondary Electron detection at $3.02 \mathrm{kV}$.

639 For transmission electron microscopy, samples were fixed in $0.1 \mathrm{M}$ sodium cacodylate buffer

$640(\mathrm{pH} 7.4)$ containing $2 \%$ glutaraldehyde and $4 \%$ paraformaldehyde at room temperature for 1 hour.

641 The fixative was then replaced with cold aqueous $1 \% \mathrm{OsO}_{4}$, and the samples were allowed to

642 warm to room temperature for 2 hours, washed three times with ultrafiltered water, and stained in

$6431 \%$ uranyl acetate for 2 hours. Samples were then dehydrated in a series of ethanol washes,

644 beginning at $50 \%$, then $70 \%$ ethanol, and then moved to $4{ }^{\circ} \mathrm{C}$ overnight. They were then placed

645 in cold $95 \%$ ethanol and allowed to warm to room temperature, changed to $100 \%$ ethanol for

64615 minutes, and finally to propylene oxide (PO) for 15 minutes. Samples were next incubated

647 with sequential EMbed-812 resin (EMS, 14120):PO mixtures of 1:2, 1:1, and 2:1 for 2 hours each

648 and stored overnight in 2:1 resin:PO. The samples were then placed into 100\% EMbed-812 resin

649 for 4 hours, moved into molds with fresh resin, orientated and warmed to $65^{\circ} \mathrm{C}$ overnight. Sections

650 were taken between 75 and $90 \mathrm{~nm}$, picked up on formvar/carbon-coated slot Cu grids, stained for

65140 seconds in $3.5 \%$ uranyl acetate in $50 \%$ acetone, followed by staining in $0.2 \%$ lead citrate for

652 six minutes. The sections were then imaged using a JEOL JEM-1400 $120 \mathrm{kV}$ instrument and a

653 Gatan Orius $8324 \mathrm{k} \times 2.6 \mathrm{k}$ digital camera with $9-\mu \mathrm{m}$ pixel size.

Microarray assay

656 Three testes from 12-week old wild-type and Hipk $4^{--}$mice were snap frozen in liquid $\mathrm{N}_{2}$, 657 and stored at $-80^{\circ} \mathrm{C}$. Samples were thawed and $50-\mathrm{mg}$ portions were immediately homogenized 658 in TRIzol, and RNA was isolated using the Direct-zol ${ }^{\mathrm{TM}}$ RNA MiniPrep Plus kit (Zymo Research, $659 \mathrm{R} 2070 \mathrm{~S})$ and stored at $-80{ }^{\circ} \mathrm{C}$. RNA profiling was then conducted in quadruplicate Mouse 660 Clariom $^{\mathrm{TM}} \mathrm{D}$ assays (Applied Biosystems, 902514), following the manufacturer's protocol. Briefly, 
$66150 \mathrm{ng}$ of each sample was used as an input into the GeneChip ${ }^{\mathrm{TM}}$ WT Plus Reagent Kit (P/N

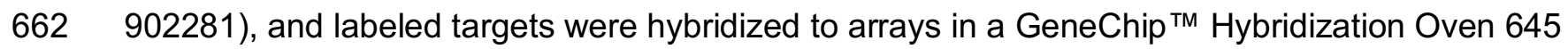

663 (00-0331). Washing and staining steps were performed on a GeneChip ${ }^{\mathrm{TM}}$ Fluidics Station 450

664 (P/N 00-0079), and arrays scanned on a GeneChip ${ }^{\mathrm{TM}}$ Scanner $30007 \mathrm{G}$ (P/N 00-210) system.

665 Data were analyzed using the Transcriptome Analysis Console 4.0 software package.

667 Retrovirus production

$668 \quad$ Murine Hipk4 and the K40S mutant genes were obtained by PCR using the primers 5'669 CAAAAAAGCAGGCTCAGCCACCATGGCCACCATCCAGTCAGAGACTG-3' and 5'-CAAGAAA 670 GCTGGGTCGTGGTGCCCTCCAACATGCTGCAG-3' for wild-type Hipk4 and 5'-TCGATCCTGA AGAACGATGCGTACCGAAGC-3' and 5'-GATGGCCACCATTTCACCTGTACTCCGAC-3' for the Hipk4-K40S mutant. pCL-ECO was purchased from Imgenex, and pBMN-I-GFP was provided by Gary Nolan. For Gateway recombination-mediated cloning, the PCR products were amplified

674 further with the primers 5'-GGGGACAAGTTTGTACAAAAAAGCAGGCTCA-3' and 5'-GGGGACC attB adapter sequences. The clones were then transferred into pDONR223 in a BP recombination

677 reaction using BP clonase II (Invitrogen) according to the manufacturer's protocols. pDONR223 678 entry constructs were next transferred to pBMN-3xFLAG-IRES-mCherry-DEST vectors using LR 679 clonase II (Invitrogen) according to the manufacturer's protocols. pBMN-HIPK4-Y175F-3xFLAG680 IRES-mCherry was later generated by site-directed mutagenesis of the wild type construct using 681 the primers 5'-CGCTATGTGAAGGAGCCTTTCATCCAGTCCCGCTTC TAC-3' and 5'-GTAGAA 682 GCGgGACTGGATGAAAGGCTCCTTCACATAGCG-3' and PfuUltra II Fusion polymerase 683 (Agilent).

684 Retroviral stocks were prepared from HEK-293T cells seeded in 10-cm tissue culture dishes $685\left(\sim 4 \times 10^{6}\right.$ cells/dish $)$ in $10 \mathrm{~mL}$ of culture medium. Approximately 18 hours post-seeding, each 10$686 \mathrm{~cm}$ dish was transfected as follows: pBMN-3xFLAG-IRES-mCherry-DEST plasmids containing 
wild type or mutant Hipk4 $(7.4 \mu \mathrm{g})$ and the pCL-ECO retrovirus packing vector $(4.4 \mu \mathrm{g})$ were

688 diluted in OMEM medium $(375 \mu \mathrm{L})$. This DNA mixture was added to $40 \mu \mathrm{L}$ Fugene® HD reagent

689 (Promega) in OMEM $(335 \mu \mathrm{L})$ and incubated at room temperature for 15 minutes, before being

690 gently added to culture medium on cells. After 24 hours, the medium was replaced with DMEM

691 containing $10 \mathrm{mM}$ HEPES ( $\mathrm{pH} 7.4$ ), 3\% fetal bovine serum, $7 \%$ calf serum, and $1 \%$ sodium

692 pyruvate. Retrovirus-containing supernatant was then collected three times at 24-hour intervals,

693 passed through a $0.45-\mu \mathrm{m}$ filter, and stored at $-80^{\circ} \mathrm{C}$.

\section{Quantitative phosphoproteomics by mass spectrometry}

To obtain peptides suitable for phospho-enrichment and mass spectrometry studies, $\mathrm{NIH}$ 3 T3 cells (passage 6) were seeded in 10-cm dishes (five per condition) at $1.5 \times 10^{6}$ cells/dish with DMEM containing $10 \%$ calf serum, $0.1 \%$ sodium pyruvate, $100 \mathrm{U} / \mathrm{mL}$ penicillin, and $0.1 \mathrm{mg} / \mathrm{mL}$ streptomycin and transduced after 12 hours with the appropriate retrovirus and polybrene $(8 \mu \mathrm{g} / \mathrm{mL})$ to achieve a multiplicity of infection $(\mathrm{MOI})>4$. After 48 hours, the culture medium was replaced with DMEM containing $0.1 \%$ CS for 4 hours, and then all dishes were transferred to a cold room, rinsed twice with ice-cold PBS, and incubated on a rocker for 10 minutes with $0.4 \mathrm{~mL}$ RIPA lysis buffer containing protease inhibitors (cOmplete ${ }^{\mathrm{TM}}$ tablets, Roche) and phosphatase inhibitors (PhosSTOP ${ }^{\text {TM }}$ tablets, Roche; 0.2 mM PMSF, Sigma). Cells were manually scraped off of each dish, and the suspensions were transferred to 15-mL Falcon tubes on ice. Cells were sonicated for 30 seconds on ice, and the lysates were cleared by centrifugation at $14000 \mathrm{~g}$. A

707 portion of each lysate was removed for protein concentration determination and western blot 708 analyses. The remaining protein lysates were precipitated with $14 \mathrm{~mL}$ of cold acetone at $-80{ }^{\circ} \mathrm{C}$.

709 Precipitates were pelleted, the supernatant was thoroughly removed, and proteins were 710 resolubilized with $2.0 \mathrm{~mL}$ of $8.0 \mathrm{M}$ urea, $50 \mathrm{mM}$ sodium bicarbonate $(\mathrm{pH} 8.0)$. Samples were 711 reduced at room temperature for 30 minutes with the addition of DTT to a final concentration of $7125 \mathrm{mM}$, and then alkylated in the dark using acrylamide at a final concentration of $10 \mathrm{mM}$ for 
71330 minutes. To digest proteins, samples were diluted to $1 \mathrm{M}$ urea with $50 \mathrm{mM}$ sodium bicarbonate

$714(\mathrm{pH}$ 8.0), Protease Max Surfactant (Promega, V2072) was added to a final concentration of

$7150.03 \%$, and a Trypsin-LysC protease mix (Promega, V5073) was added at $\sim 1: 40$ ratio to the

716 protein concentration of each sample. Samples were incubated at $37^{\circ} \mathrm{C}$ for 14 hours.

717 The digests were acidified to $\mathrm{pH} 3.5$ with formic acid and incubated for 15 minutes at $37^{\circ} \mathrm{C}$

718 to break down surfactants. Peptides were purified using Oasis HLB columns (3 mL) (Waters Co.,

719 WAT094226) according to the manufacturer's protocol, lyophilized overnight, and resolubilized in

720 a $4: 1$ acetonitrile $/ \mathrm{H}_{2} \mathrm{O}$ mixture containing $0.1 \%$ trifluoracetic acid. Peptide concentrations were

721 determined using the Pierce Peptide Quantification Colorimetric Assay. Samples were adjusted

722 to $1.0 \mathrm{mg} / \mathrm{mL}$ by adding a $4: 1$ acetonitrile/ $\mathrm{H}_{2} \mathrm{O}$ mixture containing $0.2 \%$ trifluoracetic acid, and

$72320 \mu \mathrm{L}$ of the resulting solution was saved for "total peptide" mass spectrometry runs. A standard

724 set of phosphopeptides (4 pmol/peptide sample, MS PhosphoMix1 Light, Sigma, MSPL1) were

725 spiked into each sample. Phosphopeptide-enrichment steps were performed using a 1:1 mix of

726 MagReSyn® Ti-IMAC and MagReSyn ${ }^{\circledR} \mathrm{TiO}_{2}$ resins (ReSyn, MR-TIM005 and MR-TID005)

727 according to the manufacturer's protocols. The eluted peptides were acidified to $\mathrm{pH} 2.5$ with $10 \%$

728 TFA in $\mathrm{H}_{2} \mathrm{O}$ and reduced to a volume of 5-10 $\mu \mathrm{L}$ using a SpeedVac concentrator. The samples

729 were further enriched for hydrophilic peptides by purification through graphite spin columns

730 (Pierce, 88302) according to the manufacturer's protocol. Samples were adjusted to $\mathrm{pH} \sim 8.0$ with

731 a $100 \mathrm{mM}$ triethylammonium bicarbonate solution and dried on a SpeedVac concentrator. The

732 peptides from different conditions were then isobarically labeled using the TMT-6plex kit (Pierce,

733 90061) according to the manufacturer's protocols and pooled together. Finally, these pooled

734 peptide samples were fractionated into six fractions using a high-pH reversed-phase peptide

735 fractionation kit (Pierce, 84868, lot RF231823B), and each fraction was run on a Thermo Orbitrap

736 Fusion Tribrid for LC-MS/MS analysis.

737 Peptides were identified using SEQUEST software, and individual species were removed

738 from the analysis if they: (1) had a false discovery rate was above 1\%; (2) were contaminating 
739 peptides (i.e. bovine or human); (3) were not phosphorylated. The signal intensities were then

740 normalized for each TMT channel, based on the sum of the signals detected for the standard

741 phosphopeptide mix that had been spiked into each sample prior to the phospho-enrichment step.

742 Peptides that were identified as having the same phosphorylation state were combined,

743 converging on 6,947 phosphosites with their associated A scores. The fraction of individual TMT

744 signals relative to the total intensity for each peptide was then determined.

Immunofluorescence studies

To determine the effects of Hipk4 overexpression in NIH-3T3 cells, the fibroblasts were seeded into 6-well plates at a density of 150,000 cells/well and infected 18 hours later with the appropriate retrovirus at an $\mathrm{MOI}$ of $\sim 4$. One day after infection, cells were reseeded into 24 -well plates containing poly-D-lysine-coated $12-\mathrm{mm}$ glass coverslips and cultured for 24 hours in DMEM containing $10 \%$ calf serum, $100 \mathrm{U} / \mathrm{mL}$ penicillin, and $0.1 \mathrm{mg} / \mathrm{mL}$ streptomycin. The cells were then treated with DMEM containing $0.5 \%$ calf serum and the antibiotics for an additional 24 hours, fixed in PBS containing 4\% paraformaldehyde for 10 minutes at room temperature, and washed $3 \times 5$ minutes with PBS. The cells were permeabilized with PBS containing $0.3 \%$ Triton $\mathrm{X}-100$ for 5 minutes and blocked for 2 hours at $4{ }^{\circ} \mathrm{C}$ in PBS containing $2 \%$ BSA and $0.1 \%$ Triton $\mathrm{X}-100$. The cells were then incubated for 1 hour at room temperature with primary antibodies (1:200 dilution in blocking buffer), washed $3 \times 5$ minutes with PBS, incubated for 30 minutes with the appropriate secondary antibodies (1:400 dilution in blocking buffer) and/or Alexa Fluor ${ }^{\mathrm{TM}}-647$ conjugated phalloidin (1:400, Invitrogen, A22287, lot 1884190), and washed twice more with PBS.

Nuclei were stained with DAPI, and the coverslips were rinsed briefly in water and mounted onto 761 slides using Prolong Gold Antifade reagent (Invitrogen). Immunofluorescence imaging of testis sections was conducted using cryosections of fresh

763 frozen tissue (10 $\mu \mathrm{m}$ thick). Once sectioned, samples were fixed on the slides with PBS containing $7644 \%$ paraformaldehyde for 30 minutes at room temperature, permeabilized with PBS containing 
$1 \%$ Triton-X-100 for 15 minutes, blocked with PBS containing 2\% BSA and $0.01 \%$ Triton X-100, incubated with primary antibody ( $10 \mu \mathrm{g} / \mathrm{mL}$ in blocking buffer) for 2 hours, washed three times with PBS containing $0.01 \%$ Triton X-100, incubated with secondary antibody (1:400 dilution in blocking buffer) along with either Alexa Fluor ${ }^{\mathrm{TM}}$-conjugated phalloidin (1:400) or fluorescently labeled anti-EB3 (1:100), for 30-45 minutes, washed three times with PBS containing 0.01\% Triton X-100, and mounted with coverslips with Prolong Gold with DAPI.

774 Worthington Biochemical, LS004194, lot SF8B18091A) and DNase I (1.0 mg/mL) were added, 775 and tubules were gently separated and incubated at $37^{\circ} \mathrm{C}$ for 15 minutes. The tubules were then 776 transferred to DMEM, cut into small fragments using fine scissors, and incubated with trypsin 777 (2.0 mg/mL, Worthington Biochemical, LS003702) and DNase I $(2.0 \mathrm{mg} / \mathrm{mL})$ in DMEM for 77820 minutes at $37^{\circ} \mathrm{C}$ with vigorous physical mixing every 4 minutes using a plastic transfer pipette. 779 BSA was added to stop enzymatic digestion, and the cells were pelleted at $400 \mathrm{~g}$ for 10 minutes 780 at $4{ }^{\circ} \mathrm{C}$. The cells were thoroughly resuspended in PBS containing $1 \mathrm{mg} / \mathrm{mL}$ polyvinyl alcohol 781 (PVA) and carefully loaded onto a gradient column of BSA in DMEM containing HEPES buffer 782 (10 mM, pH 7.4). The columns contained $25-\mathrm{mL}$ zones of $4 \%, 3 \%, 2 \%$, and $1 \%$ BSA. After 4 hours 783 of gravity sedimentation, fractions of cells were collected, and those containing round, elongating, 784 and condensed spermatids were combined and pelleted at $400 \mathrm{~g}$. Cells were suspended in the 785 PBS/PVA buffer, pelleted, suspended in a hypotonic sucrose solution (20 mM HEPES, $50 \mathrm{mM}$ 786 sucrose, $17 \mathrm{mM}$ sodium citrate) for 10 minutes, pelleted, and then fixed in PBS containing 4\% 787 paraformaldehyde at room temperature for 15 minutes. The cells were then permeabilized with 788 PBS containing $1 \mathrm{mg} / \mathrm{mL}$ PVA and $1.0 \%$ Triton X-100, blocked with PBS containing $2 \%$ BSA and $7891 \mathrm{mg} / \mathrm{mL}$ PVA, and incubated with the appropriate primary antibody (1:50 dilution in blocking 
791 containing $0.01 \%$ Triton $\mathrm{X}-100$ and $1 \mathrm{mg} / \mathrm{mL}$ PVA at room temperature, the cells were incubated

792 with the appropriate fluorescently labeled secondary antibody (1:400 dilution in blocking buffer)

793 along with FITC-PNA (1:1000), Alexa Fluor ${ }^{\mathrm{TM}}-647$ conjugated phalloidin (1:400), and/or

794 fluorescently labeled anti-EB3 (1:200) for colocalization) for 30-45 minutes, washed once,

795 incubated with PBS/PVA buffer containing Hoechst 33342 dye for 10 minutes at room

796 temperature, washed once more, and mounted on microscope slides using Vectashield Vibrance

797 medium.

798 All fluorescence imaging was performed using a Zeiss LSM 700 or 800 confocal microscope 799 equipped with a $63 x$ oil-immersion objective. ImageJ software $(\mathrm{NIH})$ was used to create 800 maximum-intensity Z-stack projections, and Photoshop CS6 (Adobe) was used to crop images 801 and adjust fluorescence intensity levels.

802

\section{SUPPLEMENTAL DATA}

$804 \quad$ Figure 2 - Figure supplement 1

805 Figure 2 - Figure supplement 2

$806 \quad$ Figure 3 - Figure supplement 1

$807 \quad$ Figure 3 - Figure supplement 2

808 Figure 4 - Figure supplement 1

$809 \quad$ Figure 4 - Figure supplement 2

$810 \quad$ Figure 4 - Figure supplement 3

$811 \quad$ Figure 5 - Source data 1

812 Figure 6 - Source data 1

\section{ACKNOWLEDGMENTS}

815 This work was supported by R21 HD78385 (J.K.C.), a Male Contraceptive Initiative 816 Research Grant (J.K.C.), and postdoctoral fellowships from the American Cancer Society (J.A.C.) 
and the Male Contraceptive Initiative (J.A.C.). We gratefully acknowledge Chris Adams and Ryan

818 Lieb (Stanford University Mass Spectrometry Core) for critical help with mass spectrometry 819 experiments, and Lydia-Marie Joubert (Cell Sciences Imaging Facility, Stanford University) for 820 providing training related to our scanning electron microscopy experiments. We are also indebted 821 to Pablo Visconti (University of Massachusetts), Moira O’Bryan (Monash University), George 822 Gerton (University of Pennsylvania), and Michael Eisenberg (Stanford University) for their 823 thoughtful discussions and protocols. Antibody reagents were kindly provided by Christophe 824 Arnoult (Université Grenoble Alpes), Manfred Alsheimer (University of Würzburg), and Arnoud 825 Sonnenberg (Universiteit Leiden). John Higgins (Stanford University) supplied human testis sections. The electron microscopy studies were supported, in part, by ARRA Award Number 1S10RR026780-01 from the National Center for Research Resources (NCRR).

\section{AUTHOR CONTRIBUTIONS}

J.A.C. designed and performed the experiments, analyzed the data, and wrote the paper. P.G.R. performed the qPCR and microarray assays. Z.J.H. performed animal husbandry, in situ hybridization, immunofluorescence, and SEM experiments. J.E.E. analyzed mass spectrometry data, J.J.P. prepared samples for TEM imaging, B.B. performed sperm concentration and percent motility assays. Y.L, J.L., and H.F. performed IVF and ICSI experiments and assisted with spermoocyte binding assays. J.K.C. designed the experiments, analyzed the data, and wrote the paper.

\section{AUTHOR COMPETING INTERESTS}

J.A.C. has served as a consultant for Vibliome Therapeutics, which is developing small840 molecule inhibitors of HIPK4 and other kinases, and he is now a Principal Scientist at the 841 company. J. K. C. serves on the Scientific Advisory Board for Vibliome Therapeutics. 


\section{REFERENCES}

845 Abou-haila, A. \& Tulsiani, D.R. 2009. Signal transduction pathways that regulate sperm capacitation and the acrosome reaction. Archives of Biochemistry and Biophysics 485: 7281. DOI: https://doi.org/10.1016/i.abb.2009.02.003, PMID: 19217882.

Alvau, A. et al. 2016. The tyrosine kinase fer is responsible for the capacitation-associated

Arai, S. et al. 2007. Novel homeodomain-interacting protein kinase family member, hipk4, phosphorylates human $\mathrm{p} 53$ at serine https://doi.org/10.1016/j.febslet.2007.11.022, PMID: 18022393.

Austin, C.R. 1951. Observations on the penetration of the sperm in the mammalian egg. Australian Journal of Scientific Research. Series B 4: 581-596. PMID: 14895481.

Behnen, M. et al. 2009. Testis-expressed profilins 3 and 4 show distinct functional characteristics and localize in the acroplaxome-manchette complex in spermatids. BMC Cell Biology 10: 34. DOI: https://doi.org/10.1186/1471-2121-10-34, PMID: 19419568.

Berruti, G. \& Paiardi, C. 2011. Acrosome biogenesis: Revisiting old questions to yield new insights. Spermatogenesis 1: 95-98. DOI: https://doi.org/10.4161/spmg.1.2.16820, PMID: 22319656.

Castillo, J. et al. 2019. Human testis phosphoproteome reveals kinases as potential targets in spermatogenesis and testicular cancer. Molecular \& Cellular Proteomics 18: S132-S144. DOI: https://doi.org/10.1074/mcp.RA118.001278, PMID: 30683686.

Chang, M.C. 1951. Fertilizing capacity of spermatozoa deposited into the fallopian tubes. Nature 168: 697-698. DOI: https://doi.org/10.1038/168697b0, PMID: 14882325.

Craig, A.W., Zirngibl, R., Williams, K., Cole, L.A. \& Greer, P.A. 2001. Mice devoid of fer proteintyrosine kinase activity are viable and fertile but display reduced cortactin phosphorylation. 
871 de Boer, P., de Vries, M. \& Ramos, L. 2015. A mutation study of sperm head shape and motility 872 in the mouse: Lessons for the clinic. Andrology 3: 174-202. DOI: https://doi.org/10.1111/andr.300, PMID: 25511638.

874 Di Vona, C. et al. 2015. Chromatin-wide profiling of dyrk1a reveals a role as a gene-specific rna polymerase ii ctd kinase. Molecular Cell 57: 506-520. DOI: https://doi.org/10.1016/j.molcel.2014.12.026, PMID: 25620562.

877 Escalier, D., Silvius, D. \& Xu, X. 2003. Spermatogenesis of mice lacking ck2alpha': Failure of germ cell survival and characteristic modifications of the spermatid nucleus. Molecular Reproduction and Development 66: 190-201. DOI: https://doi.org/10.1002/mrd.10346,

Fujihara, Y., Oji, A., Larasati, T., Kojima-Kita, K. \& Ikawa, M. 2017. Human globozoospermiarelated gene spata16 is required for sperm formation revealed by crispr/cas9-mediated mouse models. International Journal of Molecular Sciences 18: DOI:

Fujihara, Y. et al. 2012. Spaca1-deficient male mice are infertile with abnormally shaped sperm heads reminiscent of globozoospermia. Development 139: 3583-3589. DOI: https://doi.org/10.1242/dev.081778, PMID: 22949614.

Geyer, C.B. et al. 2009. A missense mutation in the capza3 gene and disruption of f-actin organization in spermatids of repro32 infertile male mice. Developmental Biology 330: 142152. DOI: https://doi.org/10.1016/j.ydbio.2009.03.020, PMID: 19341723. involves different polarization of two novel linc complexes. PLoS One 5: e12072. DOI: https://doi.org/10.1371/journal.pone.0012072, PMID: 20711465. 
894 Green, C.D. et al. 2018. A comprehensive roadmap of murine spermatogenesis defined by single-cell rna-seq. Developmental Cell 46: 651-667 e610. DOI: https://doi.org/10.1016/j.devcel.2018.07.025, PMID: 30146481.

Han, F. et al. 2017. Globozoospermia and lack of acrosome formation in gm130-deficient mice. Cell Death \& Disease 8: e2532. DOI: https://doi.org/10.1038/cddis.2016.414, PMID: 28055014 .

He, Q. et al. 2010. Characterization of human homeodomain-interacting protein kinase 4 (hipk4) as a unique member of the hipk family. Molecular and Cellular Pharmacology 2: 61-68. PMID: 20508833.

Hirohashi, N. 2016. Site of mammalian sperm acrosome reaction. Advances in Anatomy, Embryology and Cell Biology 220: 145-158. DOI: https://doi.org/10.1007/978-3-319-305677 8, PMID: 27194354.

Inoue, N., Ikawa, M., Isotani, A. \& Okabe, M. 2005. The immunoglobulin superfamily protein izumo is required for sperm to fuse with eggs. Nature 434: 234-238. DOI: https://doi.org/10.1038/nature03362, PMID: 15759005.

Jha, K.N. et al. 2013. Heat shock protein 90 functions to stabilize and activate the testis-specific serine/threonine kinases, a family of kinases essential for male fertility. Journal of Biological Chemistry 288: 16308-16320. DOI: https://doi.org/10.1074/ibc.M112.400978, PMID: 23599433.

916 Kang-Decker, N., Mantchev, G.T., Juneja, S.C., McNiven, M.A. \& van Deursen, J.M. 2001. Lack 
Keshet, E., Itin, A., Fischman, K. \& Nir, U. 1990. The testis-specific transcript (fert) of the tyrosine kinase fer is expressed during spermatogenesis in a stage-specific manner. Molecular and Cellular Biology 10: 5021-5025. DOI: https://doi.org/10.1128/mcb.10.9.5021, PMID:

Kierszenbaum, A.L. 2000. Fusion of membranes during the acrosome reaction: $A$ tale of two snares. Molecular Reproduction and Development

57: $\quad 309-310$

DOI: https://doi.org/10.1002/1098-2795(200012)57:4<309::AID-MRD1>3.0.CO;2-W, PMID: 11066058.

Kierszenbaum, A.L., Rivkin, E. \& Tres, L.L. 2003. Acroplaxome, an f-actin-keratin-containing plate, anchors the acrosome to the nucleus during shaping of the spermatid head. Molecular Biology of the Cell 14: 4628-4640. DOI: https://doi.org/10.1091/mbc.e03-04-0226, PMID:

Kierszenbaum, A.L., Rivkin, E. \& Tres, L.L. 2003. The actin-based motor myosin va is a component of the acroplaxome, an acrosome-nuclear envelope junctional plate, and of manchette-associated vesicles. Cytogenetic and Genome Research 103: 337-344. DOI: https://doi.org/10.1159/000076822, PMID: 15051957.

Kierszenbaum, A.L., Rivkin, E. \& Tres, L.L. 2007. Molecular biology of sperm head shaping. Society for Reproduction and Fertility Supplement 65: 33-43. PMID: 17644953.

Kierszenbaum, A.L., Rivkin, E. \& Tres, L.L. 2008. Expression of fer testis (fert) tyrosine kinase transcript variants and distribution sites of fert during the development of the acrosome-

941 Kierszenbaum, A.L., Rivkin, E. \& Tres, L.L. 2011. Cytoskeletal track selection during cargo acroplaxome-manchette complex in rat spermatids. Developmental Dynamics 237: 38823891. DOI: https://doi.org/10.1002/dvdy.21789, PMID: 18985748. transport in spermatids is relevant to male fertility. Spermatogenesis 1: 221-230. DOI: https://doi.org/10.4161/spmg.1.3.18018, PMID: 22319670. 
944 Kierszenbaum, A.L. \& Tres, L.L. 2004. The acrosome-acroplaxome-manchette complex and the shaping of the spermatid head. Archives of Histology and Cytology 67: 271-284. PMID:

Kierszenbaum, A.L., Tres, L.L., Rivkin, E., Kang-Decker, N. \& van Deursen, J.M. 2004. The acroplaxome is the docking site of golgi-derived myosin va/rab27a/b- containing

Kim, K.S., Cha, M.C. \& Gerton, G.L. 2001. Mouse sperm protein sp56 is a component of the acrosomal matrix. Biology of Reproduction

64: $\quad 36-43$.

DOI: https://doi.org/10.1095/biolreprod64.1.36, PMID: 11133656.

Kim, Y.H., Choi, C.Y., Lee, S.J., Conti, M.A. \& Kim, Y. 1998. Homeodomain-interacting protein kinases, a novel family of co-repressors for homeodomain transcription factors. Journal of Biological Chemistry 273: 25875-25879. DOI: https://doi.org/10.1074/jbc.273.40.25875, PMID: 9748262.

Kueng, P. et al. 1997. A novel family of serine/threonine kinases participating in spermiogenesis. Journal of Cell Biology 139: 1851-1859. DOI: https://doi.org/10.1083/jcb.139.7.1851, PMID: 9412477.

Lehti, M.S. \& Sironen, A. 2016. Formation and function of the manchette and flagellum during spermatogenesis. Reproduction 151: R43-54. DOI: https://doi.org/10.1530/REP-15-0310, PMID: 26792866.

Li, W. et al. 2015. A meig1/pacrg complex in the manchette is essential for building the sperm flagella. Development 142: 921-930. DOI: https://doi.org/10.1242/dev.119834, PMID: 
$\mathrm{Li}, \mathrm{Y}$. et al. 2017. A nonsense mutation in ccdc62 gene is responsible for spermiogenesis defects and male infertility in repro29/repro29 mice. Biology of Reproduction 96: 587-597. DOI: https://doi.org/10.1095/biolreprod.116.141408, PMID: 28339613.

Lin, Y. \& Kan, F.W. 1996. Regionalization and redistribution of membrane phospholipids and cholesterol in mouse spermatozoa during in vitro capacitation. Biology of Reproduction 55: 1133-1146. DOI: https://doi.org/10.1095/biolreprod55.5.1133, PMID: 8902227.

Litovchick, L., Florens, L.A., Swanson, S.K., Washburn, M.P. \& DeCaprio, J.A. 2011. Dyrk1a

Liu, Y. et al. 2015. Lrguk-1 is required for basal body and manchette function during spermatogenesis and male fertility. PLoS Genetics 11: e1005090. DOI: https://doi.org/10.1371/journal.pgen.1005090, PMID: 25781171.

Mao, J. et al. 2002. Regulation of gli1 transcriptional activity in the nucleus by dyrk1. Journal of Biological Chemistry 277: 35156-35161. DOI: https://doi.org/10.1074/jbc.M206743200, PMID: 12138125.

Naz, R.K. \& Rajesh, P.B. 2004. Role of tyrosine phosphorylation in sperm capacitation / acrosome reaction. Reproductive Biology and Endocrinology 2: 75 . DOI: https://doi.org/10.1186/1477-7827-2-75, PMID: 15535886.

Nozawa, Y.I., Yao, E., Gacayan, R., Xu, S.M. \& Chuang, P.T. 2014. Mammalian fused is essential for sperm head shaping and periaxonemal structure formation during spermatogenesis. Developmental Biology 388: 170-180. DOI: https://doi.org/10.1016/j.ydbio.2014.02.002, PMID: 24525297.

O'Donnell, L. 2014. Mechanisms of spermiogenesis and spermiation and how they are disturbed. Spermatogenesis 4: e979623. DOI: https://doi.org/10.4161/21565562.2014.979623, PMID: 26413397. 
994 O'Donnell, L. et al. 2012. An essential role for katanin p80 and microtubule severing in male gamete production. PLOS Genetics

8: $\quad$ e1002698.

DOI: https://doi.org/10.1371/journal.pgen.1002698, PMID: 22654669.

997 O'Rand, M.G. 1982. Modification of the sperm membrane during capacitation. Annals of the New 998 York Academy of Sciences 383: 392-404. DOI: https://doi.org/10.1111/j.17496632.1982.tb23180.x.

Pasch, E., Link, J., Beck, C., Scheuerle, S. \& Alsheimer, M. 2015. The linc complex component sun4 plays a crucial role in sperm head formation and fertility. Biology Open 4: 1792-1802. DOI: https://doi.org/10.1242/bio.015768, PMID: 26621829.

Pasek, R.C. et al. 2016. Coiled-coil domain containing 42 (ccdc42) is necessary for proper sperm development and male fertility in the mouse. Developmental Biology 412: 208-218. DOI: https://doi.org/10.1016/j.ydbio.2016.01.042, PMID: 26945718.

Pierre, V. et al. 2012. Absence of dpy19l2, a new inner nuclear membrane protein, causes globozoospermia in mice by preventing the anchoring of the acrosome to the nucleus. Development 139: 2955-2965. DOI: https://doi.org/10.1242/dev.077982, PMID: 22764053.

Rathke, C., Baarends, W.M., Awe, S. \& Renkawitz-Pohl, R. 2014. Chromatin dynamics during spermiogenesis. Biochimica et et Biophysica Acta 1839: $155-168$.

DOI: https://doi.org/10.1016/..bbagrm.2013.08.004, PMID: 24091090.

Rinaldo, C., Siepi, F., Prodosmo, A. \& Soddu, S. 2008. Hipks: Jack of all trades in basic nuclear activities. Biochimica et Biophysica Acta 1783: 2124-2129. DOI: https://doi.org/10.1016/j.bbamcr.2008.06.006, PMID: 18606197.

1016 the testis. (Cache River Press, Clearwater, FL; 1990).

1017 Sacher, F., Fritsch, M., Feldman, R.I. \& Möller, C. (2008) Hip kinase for fertility control. Eur. Pat. $1018 \quad$ Appl. EP1889626. 
1019 Schnabel, D., Ramirez, L., Gertsenstein, M., Nagy, A. \& Lomeli, H. 2005. Ectopic expression of kitd814y in spermatids of transgenic mice, interferes with sperm morphogenesis.

Sebkova, N., Ded, L., Vesela, K. \& Dvorakova-Hortova, K. 2014. Progress of sperm izumo1 Developmental Dynamics 233: 29-40. DOI: https://doi.org/10.1002/dvdy.20292, PMID:

Sepideh, J. et al. 2009. Tyrosine phosphorylation pattern in sperm proteins isolated from normospermic and teratospermic men. Journal of Reproduction \& Infertility 10: 185-191. PMID: 23926467.

Shang, P. et al. 2010. Functional transformation of the chromatoid body in mouse spermatids requires testis-specific serine/threonine kinases. Journal of Cell Science 123: 331-339. DOI: https://doi.org/10.1242/jcs.059949, PMID: 20053632.

Shang, Y. et al. 2017. Essential role for sun5 in anchoring sperm head to the tail. eLife 6: DOI: https://doi.org/10.7554/eLife.28199, PMID: 28945193.

Sosnik, J. et al. 2009. Tssk6 is required for izumo relocalization and gamete fusion in the mouse. Journal of Cell Science 122: 2741-2749. DOI: https://doi.org/10.1242/jcs.047225, PMID: 19596796.

Spiridonov, N.A. et al. 2005. Identification and characterization of sstk, a serine/threonine protein

Sprando, R.L. \& Russell, L.D. 1987. Comparative study of cytoplasmic elimination in spermatids of selected mammalian species. American Journal of Anatomy 178: 72-80. DOI: https://doi.org/10.1002/aja.1001780109, PMID: 3825964. 
1043 Stival, C. et al. 2016. Sperm capacitation and acrosome reaction in mammalian sperm. Advances in Anatomy, Embryology and Cell Biology 220: 93-106. DOI: https://doi.org/10.1007/978-3319-30567-7 5, PMID: 27194351.

1046 Tuttelmann, F., Ruckert, C. \& Ropke, A. 2018. Disorders of spermatogenesis: Perspectives for novel genetic diagnostics after 20 years of unchanged routine. Medizinsche Genetik 30: 1220. DOI: https://doi.org/10.1007/s11825-018-0181-7, PMID: 29527098.

van der Laden, J., Soppa, U. \& Becker, W. 2015. Effect of tyrosine autophosphorylation on catalytic activity and subcellular localisation of homeodomain-interacting protein kinases (hipk). Cell Communication and Signaling 13: 3. DOI: https://doi.org/10.1186/s12964-0140082-6, PMID: 25630557.

Wang, X. et al. 2016. Testis-specific serine/threonine protein kinase 4 (tssk4) phosphorylates odf2 at ser-76. Science Reports 6: 22861. DOI: https://doi.org/10.1038/srep22861, PMID: 26961893.

Wang, X. et al. 2015. Tssk4 is essential for maintaining the structural integrity of sperm flagellum. Molecular Human Reproduction 21: 136-145. DOI: https://doi.org/10.1093/molehr/gau097, PMID: 25361759.

Wong, E.W., Mruk, D.D. \& Cheng, C.Y. 2008. Biology and regulation of ectoplasmic specialization, an atypical adherens junction type, in the testis. Biochimica et Biophysica Acta 1778: 692-708. DOI: https://doi.org/10.1016/j.bbamem.2007.11.006, PMID: 18068662.

Woods, Y.L. et al. 2001. The kinase dyrk1a phosphorylates the transcription factor fkhr at ser329 in vitro, a novel in vivo phosphorylation site. Biochemical Journal 355: 597-607. DOI: https://doi.org/10.1042/bj3550597, PMID: 11311120.

Wu, J.Y. et al. 2000. Spermiogenesis and exchange of basic nuclear proteins are impaired in male germ cells lacking camk4. Nature Genetics 25: 448-452. DOI: https://doi.org/10.1038/78153, PMID: 10932193. 
1068

1069

1070

1071

1072

1073

1074

1075

1076

1077

1078

1079

1080

1081

1082

1083

1084

1085

1086

1087

1088

1089

1090

1091

1092

1093

Xiao, N. et al. 2009. Pick1 deficiency causes male infertility in mice by disrupting acrosome formation. Journal of Clinical Investigation 119: 802-812. DOI: https://doi.org/10.1172/JCI36230, PMID: 19258705.

$\mathrm{Xu}$, B. et al. 2008. Targeted deletion of tssk1 and 2 causes male infertility due to haploinsufficiency. Developmental Biology 319: 211-222. DOI: https://doi.org/10.1016/j.ydbio.2008.03.047, PMID: 18533145.

Yan, W. 2009. Male infertility caused by spermiogenic defects: Lessons from gene knockouts. Molecular and Cellular Endocrinology 306: 24-32. DOI: https://doi.org/10.1016/j.mce.2009.03.003, PMID: 19481682.

Yang, E.J., Ahn, Y.S. \& Chung, K.C. 2001. Protein kinase dyrk1 activates camp response element-binding protein during neuronal differentiation in hippocampal progenitor cells. Journal of Biological Chemistry 276: 39819-39824. DOI: https://doi.org/10.1074/jbc.M104091200, PMID: 11518709.

Yao, R. et al. 2002. Lack of acrosome formation in mice lacking a golgi protein, gopc. PNAS 99: 11211-11216. DOI: https://doi.org/10.1073/pnas.162027899, PMID: 12149515.

Yoshida, N. \& Perry, A.C. 2007. Piezo-actuated mouse intracytoplasmic sperm injection (icsi). Nature Protocols 2: 296-304. DOI: https://doi.org/10.1038/nprot.2007.7, PMID: 17406589.

Zakrzewski, P., Lenartowski, R., Redowicz, M.J., Miller, K.G. \& Lenartowska, M. 2017. Expression and localization of myosin vi in developing mouse spermatids. Histochemistry and Cell Biology 148: 445-462. DOI: https://doi.org/10.1007/s00418-017-1579-z, PMID: 28500503.

Zheng, H. et al. 2007. Lack of spem1 causes aberrant cytoplasm removal, sperm deformation, and male infertility. PNAS 104: 6852-6857. DOI: https://doi.org/10.1073/pnas.0701669104, PMID: 17426145.

Zhou, J. et al. 2009. Rim-bp3 is a manchette-associated protein essential for spermiogenesis. Development 136: 373-382. DOI: https://doi.org/10.1242/dev.030858, PMID: 19091768. 
bioRxiv preprint doi: https://doi.org/10.1101/703637; this version posted July 16,2019 . The copyright holder for this preprint (which was not certified by peer review) is the author/funder, who has granted bioRxiv a license to display the preprint in perpetuity. It is made available under aCC-BY-NC-ND 4.0 International license. 
bioRxiv preprint doi: https://doi org/10.1101/703637; this version posted July 16,2019. The copyright holder for this preprint (which was not certified by peer review) is the author/funder, who has granted bioRxiv a license to display the preprint in perpetuity. It is made available under aCC-BY-NC-ND 4.0 International license.
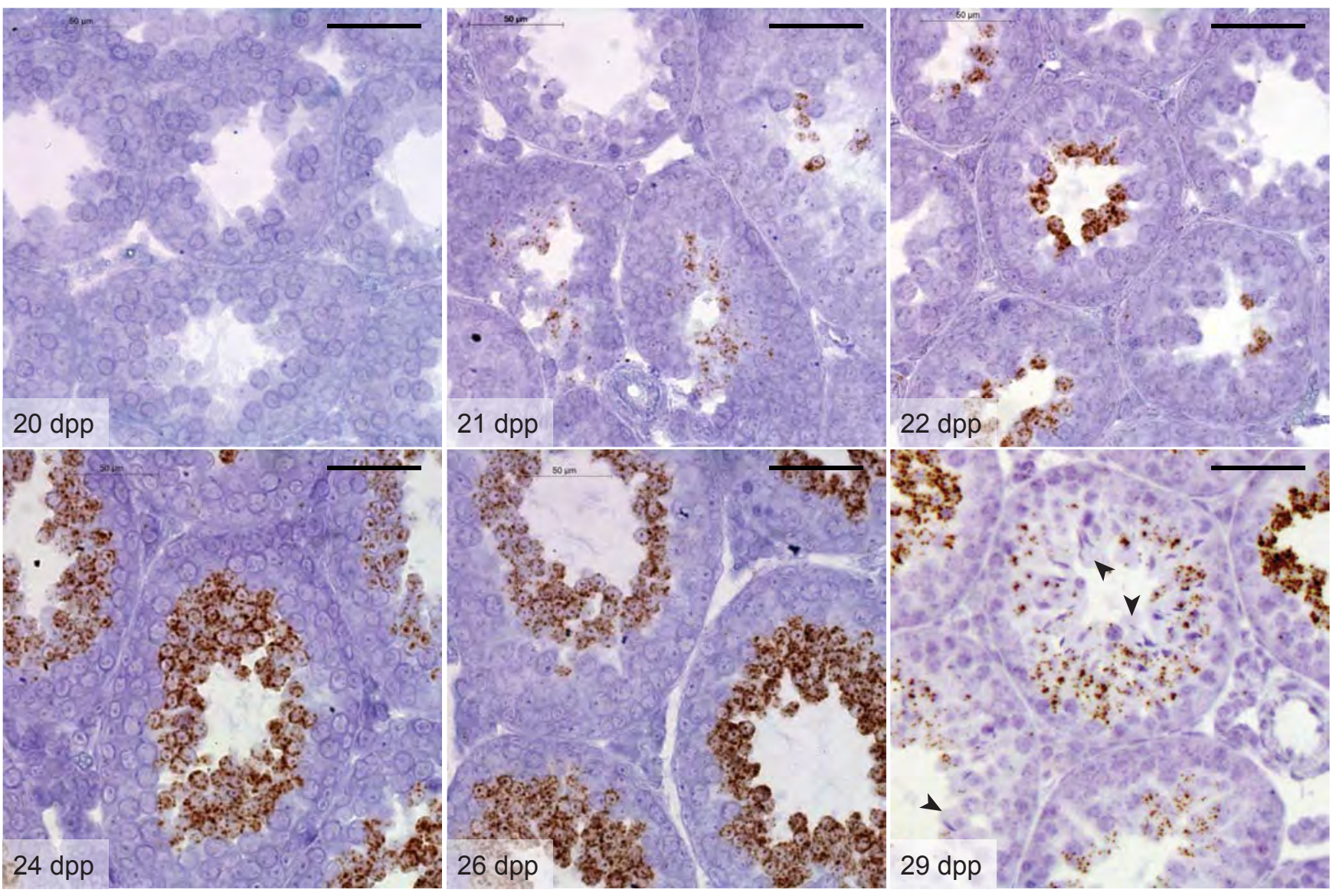

Figure 2 - figure supplement 1. Hipk4 mRNA expression during the first wave of murine spermatogenesis. Hipk4 transcript labeling by in is tu hybridia tion in formalin-fixed, paraffin-embedded testis sections from wild-type C57BL/6NJ mice of the designated ages. Arrowheads indicate the loss of Hipk4 expression in elongating spermatids circumscribing the seminiferous tubule lumen (29 dpp). Scale bars: $50 \mu \mathrm{m}$. 
bioRxiv preprint doi: https://doi.org/10.1101/703637; this version posted July 16,2019 . The copyright holder for this preprint (which was not certified by peer review) is the author/funder, who has granted bioRxiv a license to display the preprint in perpetuity. It is made available under aCC-BY-NC-ND 4.0 International license.

A WT

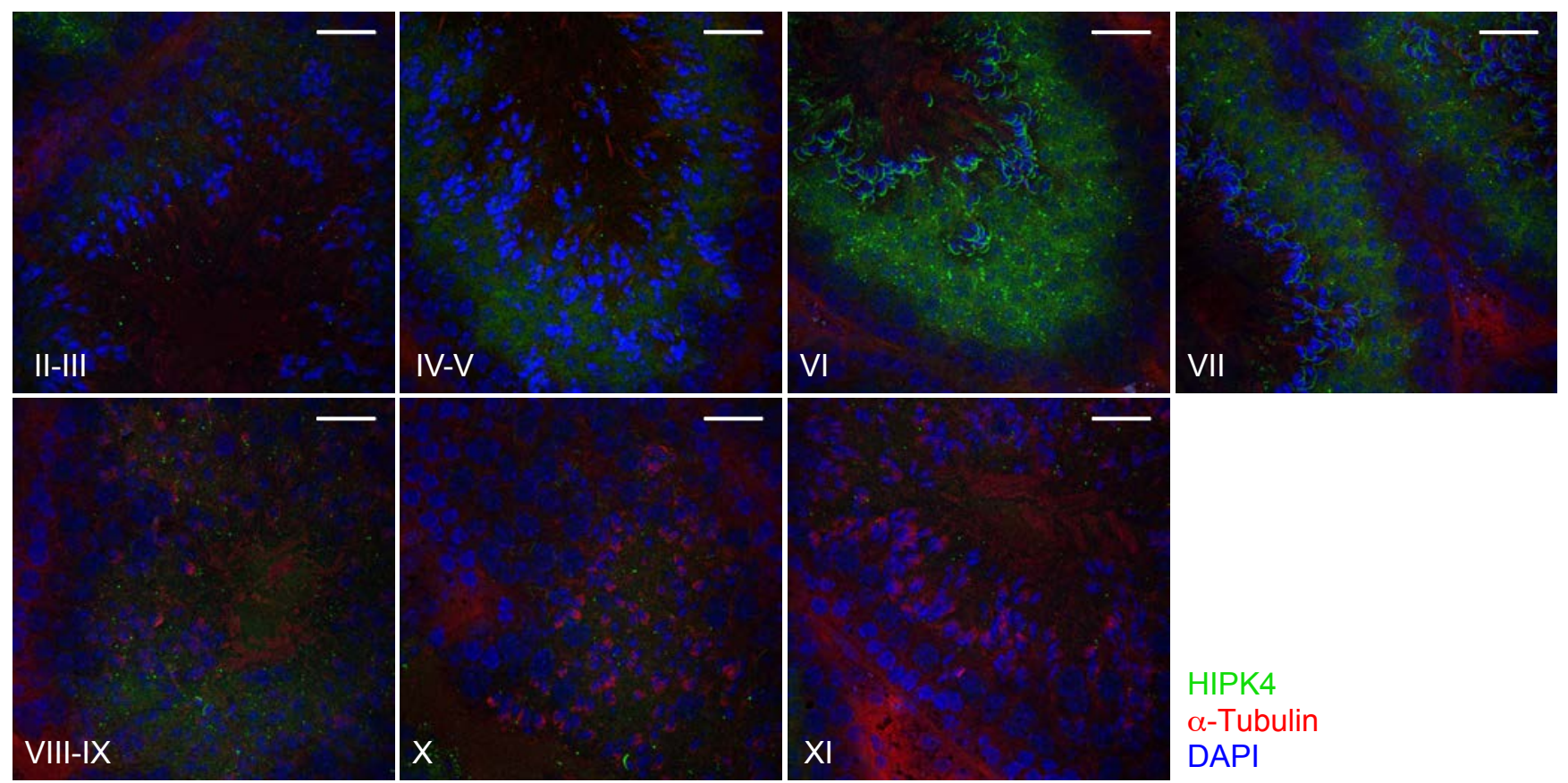

B

Hipk4 $4^{-1-}$

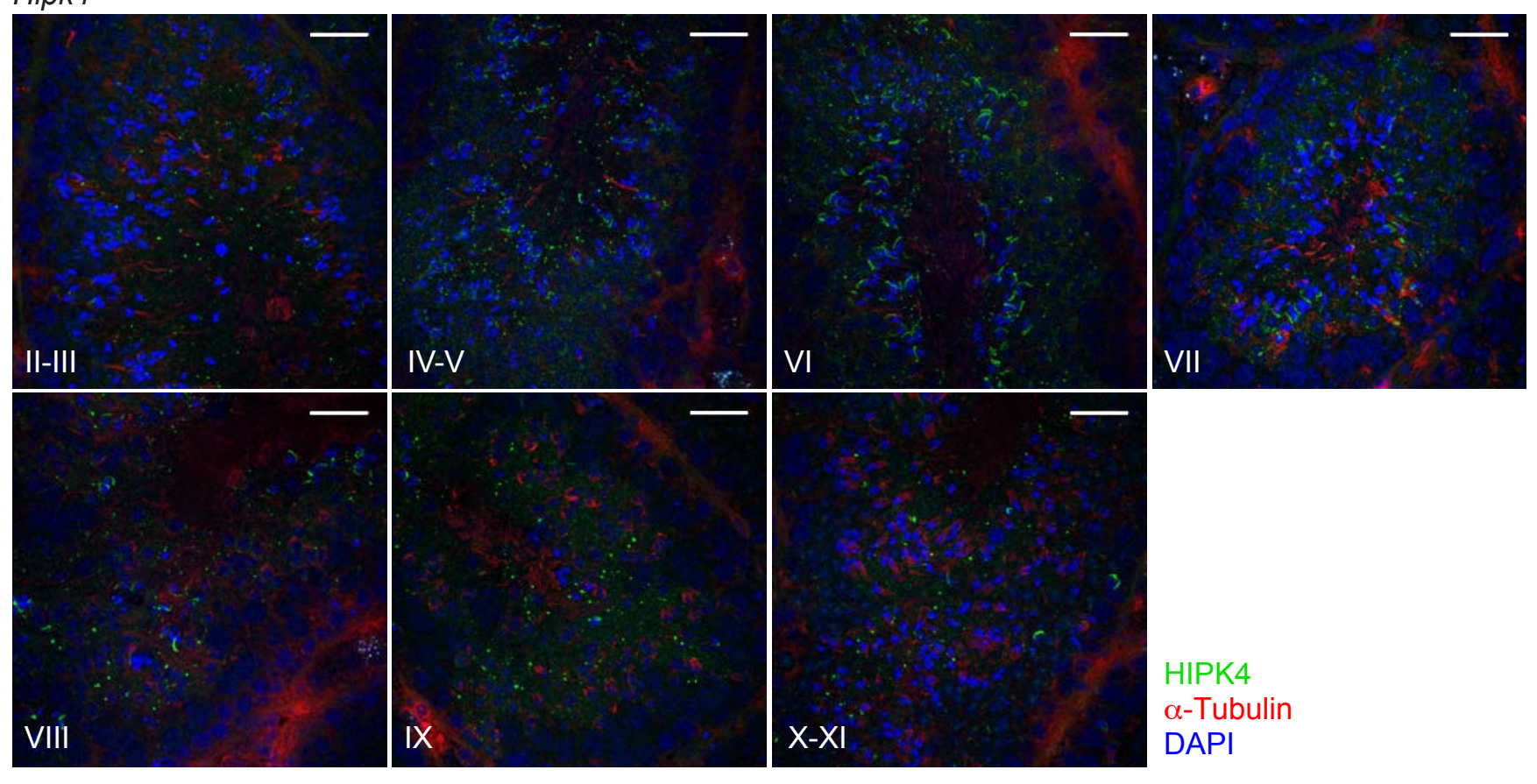

Figure 2 - figure supplement 2. HIPK4 protein expression in adult murine seminiferous tubules. (A-B) Immunofluorescence images of cryosectioned testes from adult wild-type (A) and Hipk4 knockout (B) mice. Stages for each testis section are shown. Scale bars: $20 \mu \mathrm{m}$. 
bioRxiv preprint doi: https://doi.org/10.1101/703637; this version posted July 16,2019 . The copyright holder for this preprint (which was not certified by peer review) is the author/funder, who has granted bioRxiv a license to display the preprint in perpetuity. It is made available under aCC-BY-NC-ND 4.0 International license.

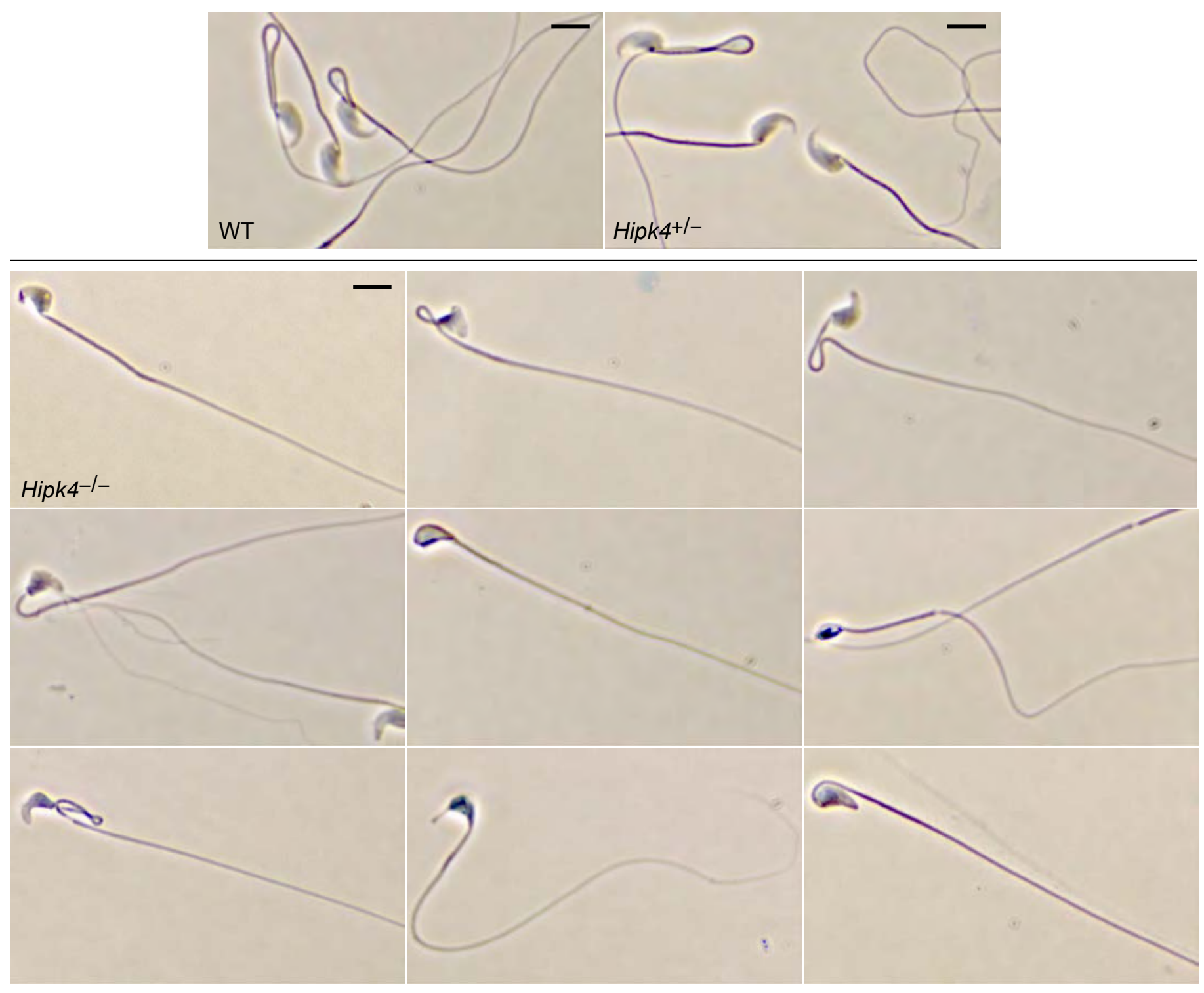

Figure 3 - figure supplement 1. Morphologies of wild-type and Hipk4 mutant sperm. Phase contrast images of sperm obtained from mice with the designated genotypes. Sperm were treated with Diff-Quik stain prior to imaging, and multiple examples of homozygous mutant sperm are shown to illustrate the range of morphological phenotypes. Scale bars: $2 \mu \mathrm{m}$. 
$\mathbf{A}$

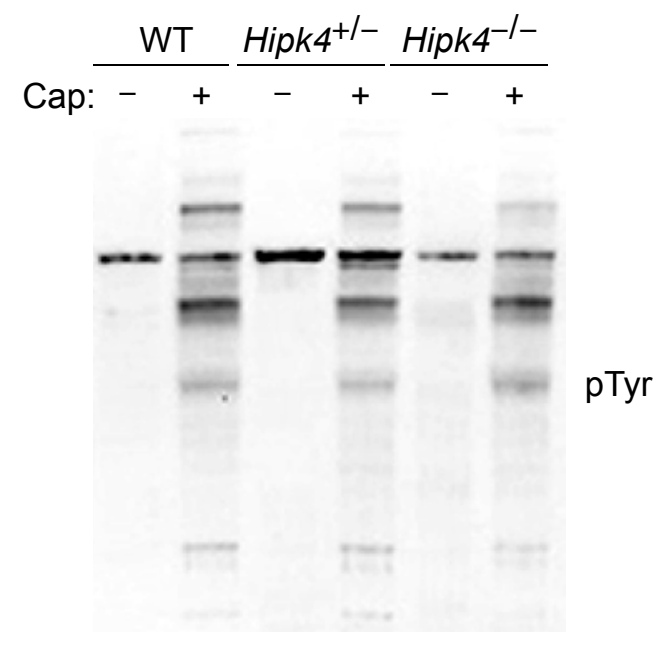

C

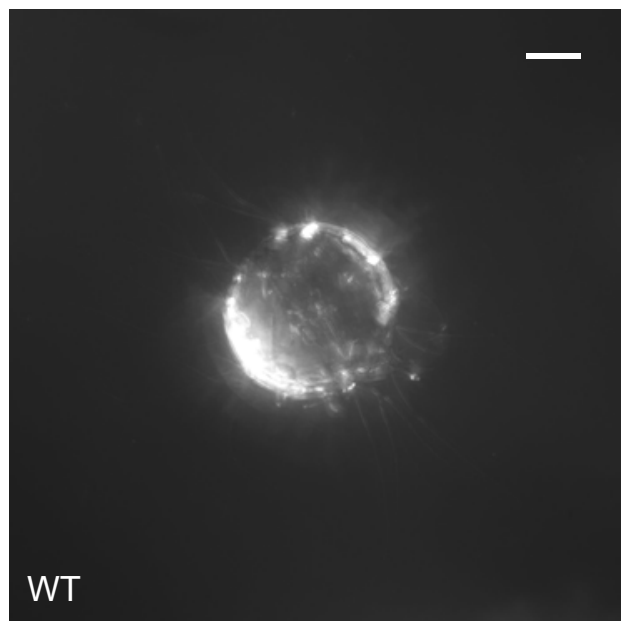

B
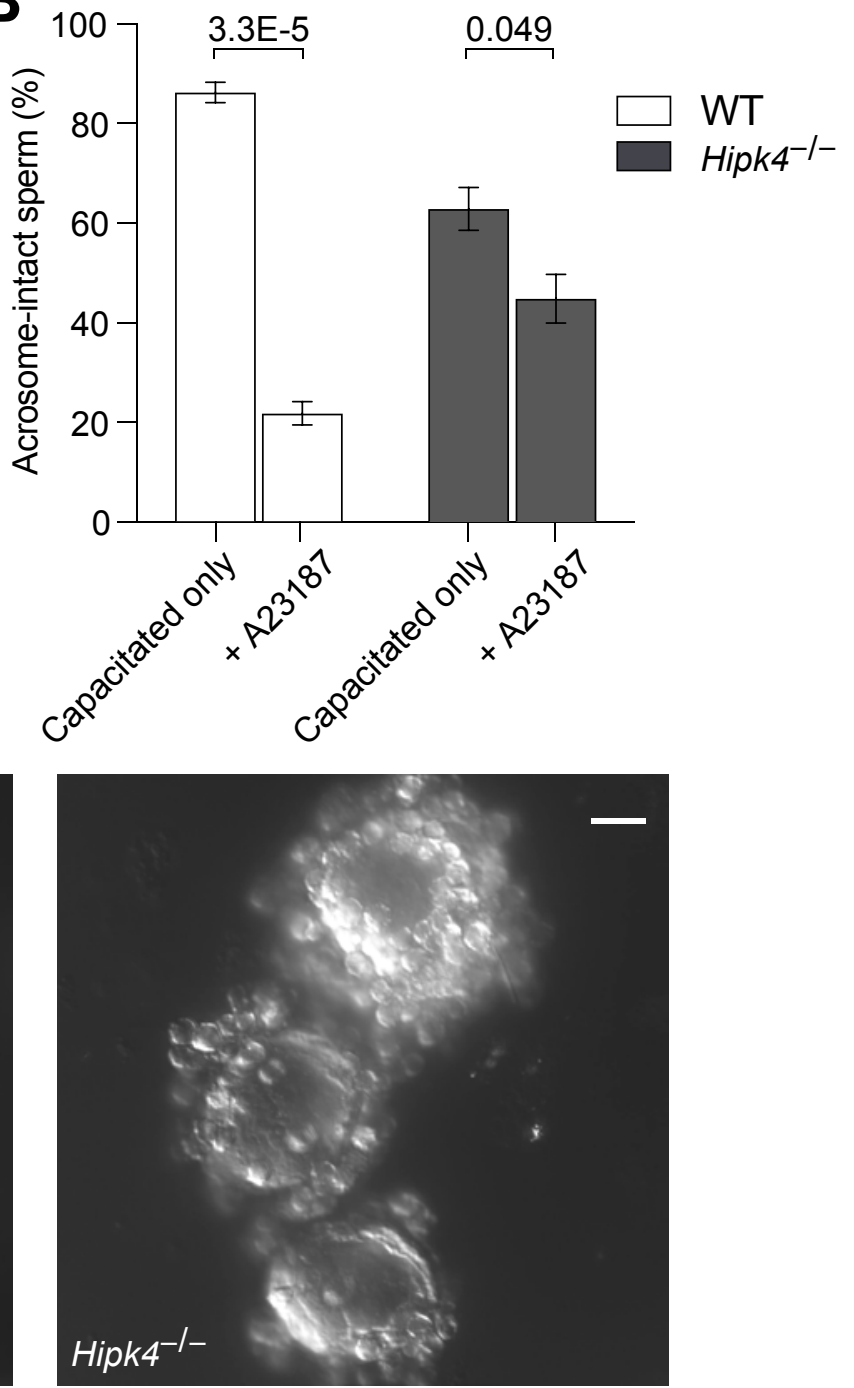

Figure 3 - figure supplement 2. HIPK4 is not essential for sperm capacitation or acrosomal exocytosis. (A) Western blot detection of soluble phosphotyrosine-containing proteins in sperm before or after capacitation with TYH medium + BSA (Cap buffer). (B) Percentage of wild-type or Hipk4 $\mathrm{k}$ ock out sperm with an acrosome after treatment with Cap buffer for 1.5 hours or after an additional 1-hour incubation with either the $\mathrm{Ca}^{2+}$ ionophore $\mathrm{A} 23187$. Data are the average of three independent experiments \pm s.e.m., and the number of sperm counted for each condition was between 213-1228. $P$-values (T-test; two-tailed, equal variance) are shown. (C) Representative differential interference contrast (DIC) images of oocytes following standard IVF conditions using wild-type or Hipk4 $\mathrm{k}$ ock ut sperm. Scale bars $=20 \mu \mathrm{m}$ 


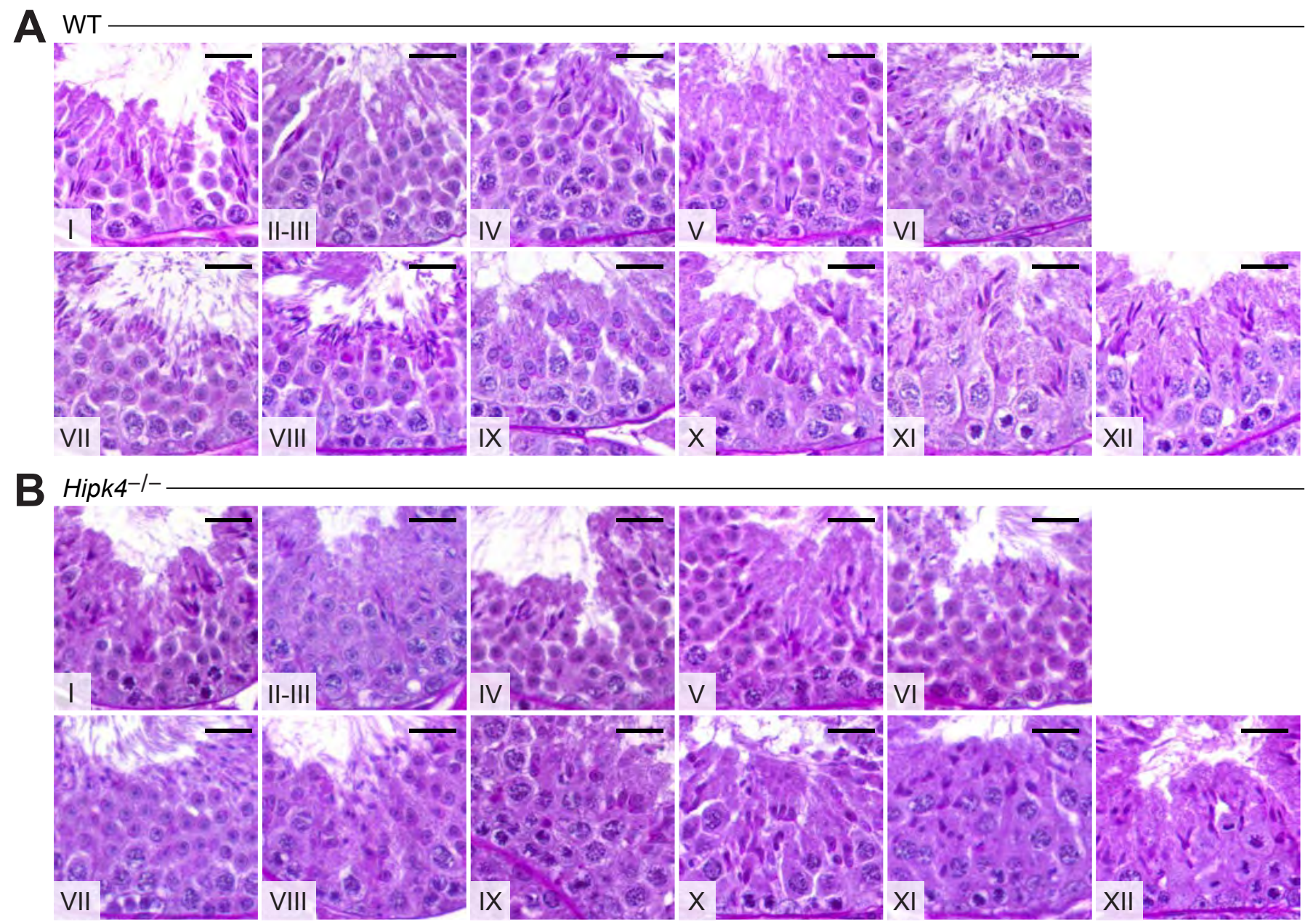

C WT
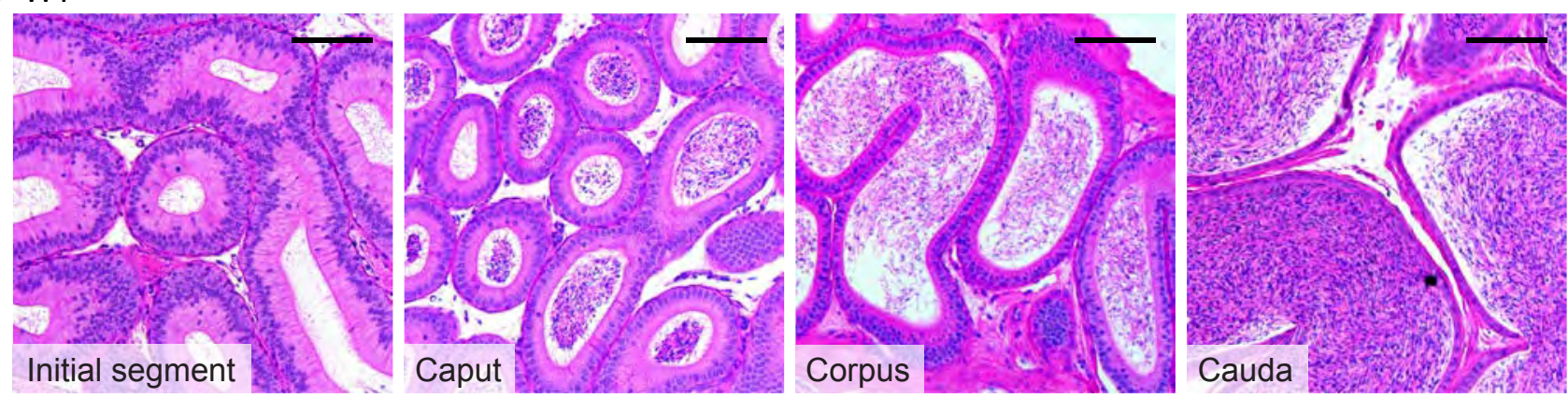

D
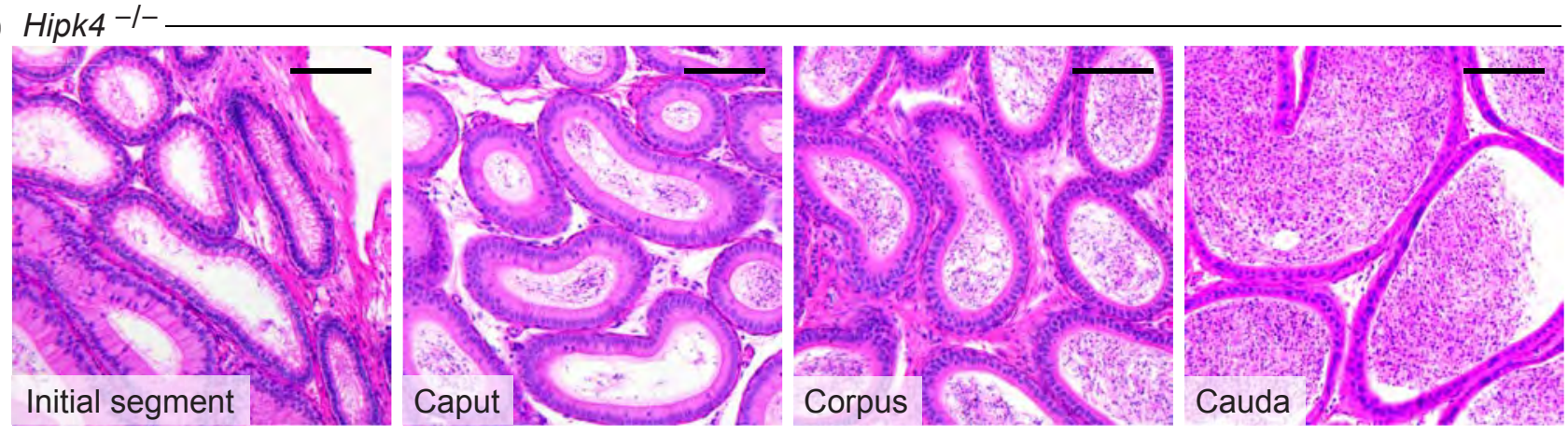

Figure 4 - figure supplement 1. Comparison of the seminiferous epithelium and epididymis of WT and Hipk4 knockout mice. (A-B) Fixed, paraffin-embedded testis sections from WT (A) and Hipk4-/- $(B)$ mice stained with PAS reagents. Stages for the tubules shown in each micrograph are indicated. (C-D) Fixed, paraffin-embedded epididymis sections from WT (C) and Hipk4-/- $(D)$ mice stained with hematoxylin and eosin. Epididymal regions associated with each section are indicated. Scale bars: $20 \mu \mathrm{m}$. 
bioRxiv preprint doi: https://doi.org/10.1101/703637; this version posted July 16 2019. The copyright holder for this preprint (which was not certified by peer review) is the author/funder, who has granted bioRxiv a license to display the preprint in perpetuity. It is made available under aCC-BY-NC-ND 4.0 International license.

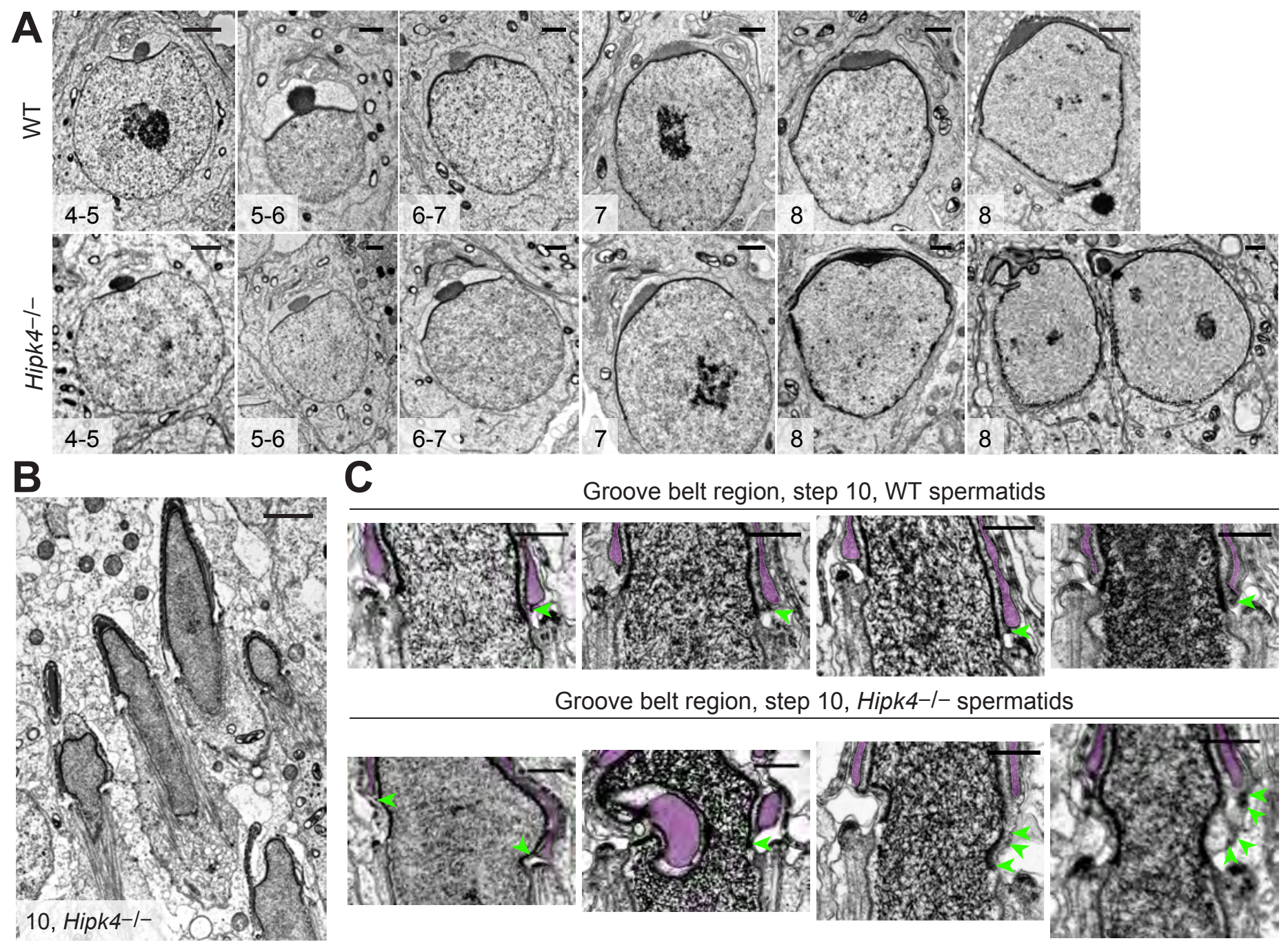

Figure 4 - figure supplement 2. HIPK4 null spermatids exhibit acrosome-acroplaxome defects. (A) TEM images of step 4-8 spermatids from adult WT and Hipk4-/- mice. (B) TEM images of step 10 Hipk $4^{-/}$spermatids. (C) Higher magnification TEM images of the groove belt region in step 10 WT and Hipk ${ }^{-1}$ - spermatids. Acrosomes are falsecolored purple, and green arrowheads label electron densities in the acroplaxome that normally are associated with the acrosome or plasma membrane. Scale bars: A, $1 \mu \mathrm{m}$; B, $2 \mu \mathrm{m}$; C, $1 \mu \mathrm{m}$. 

aCC-BY-NC-ND 4.0 International license.

A

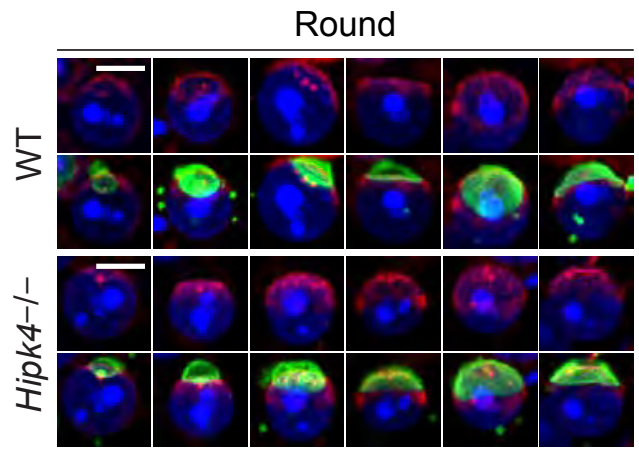

Elongating

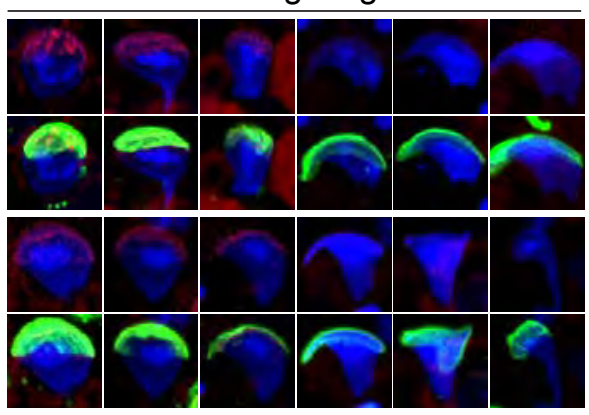

PNA Hoechst 33342

B
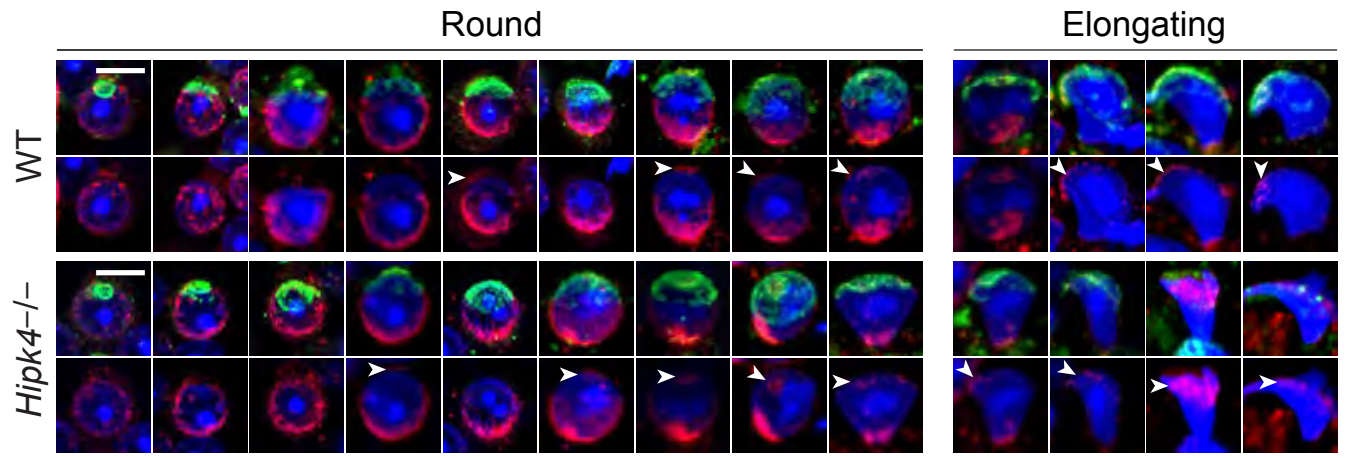

SUN1 Nesprin3 Hoechst 33342
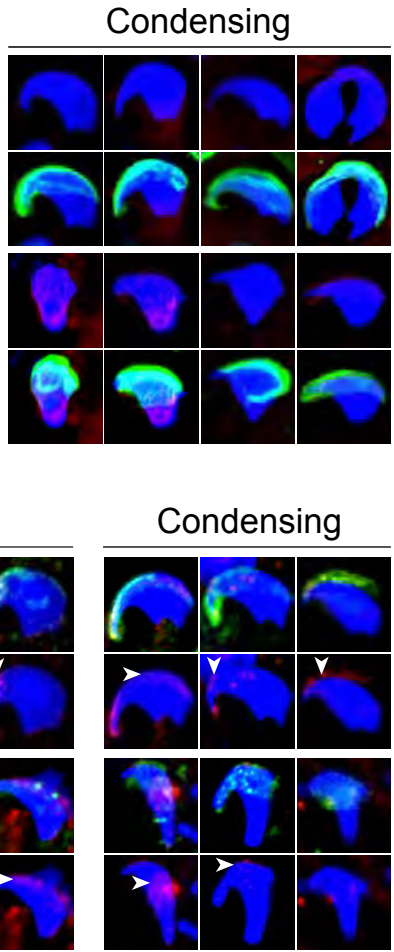

C

Manchette progression - sections of seminiferous epithelium

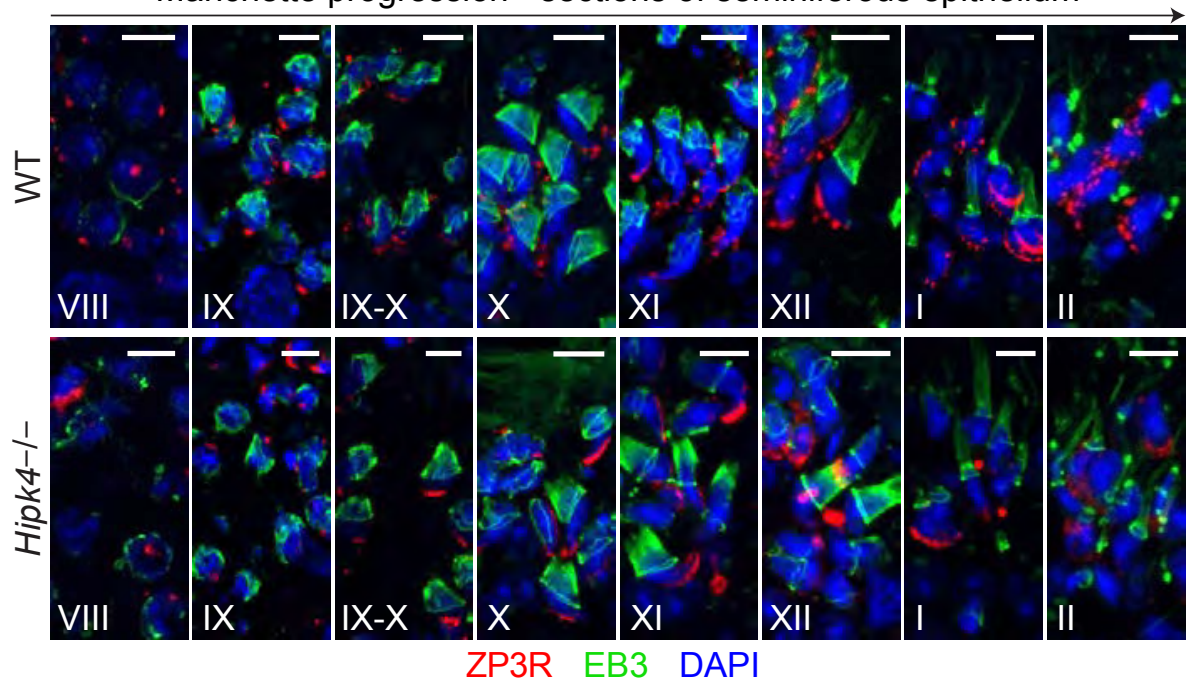

Manchette progression - dissociated spermatids
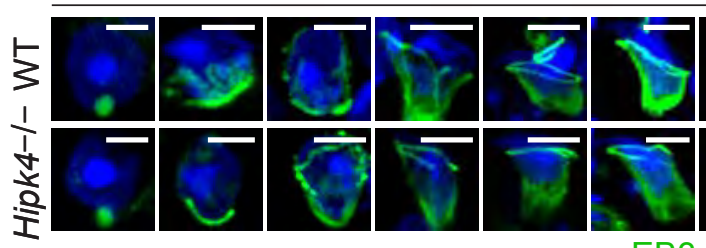

EB3 Hoechst 33342

Figure 4 - figure supplement 3. HIPK4 does not regulate the localization of anterior LINC complexes or manchette dynamics. (A) Immunofluorescence imaging of the inner nuclear membrane protein DPY19L2 (red) in dissociated spermatids. The acrosome is labeled with FITC-PNA (green), and nuclei are stained with Hoechst 33342 (blue). (B) Immunofluorescence imaging of the LINC complex proteins SUN1 (red) and nesprin3 (green) in dissociated spermatids. Nuclei are stained with Hoechst 33342 (blue). (C) Immunofluorescence imaging of EB3 (green) and ZP3R (red) in testis cryosections. Nuclei are stained with DAPI, and stages of each section are indicated. (D) Immunofluorescence imaging of EB3 (green) in dissociated spermatids. Nuclei are stained with Hoechst 33342 (blue). Scale bars: A-B (scale bars are representative for all images across the row), $2 \mu \mathrm{m} ; \mathrm{C}$, $5 \mu \mathrm{m}$; D, $2 \mu \mathrm{m}$. 